\title{
The Study of Asymptotically Fine Wrinkling in Nonlinear Elasticity using a Boundary Layer Analysis
}

\author{
James MacLaurin ${ }^{14}$, Jon Chapman ${ }^{1}$, Gareth Wyn Jones ${ }^{2}$, Tiina Roose ${ }^{3}$ \\ ${ }^{1}$ Mathematical Institute, University of Oxford 24-29 St Giles OX1 3LB Oxford UK, \\ james.maclaurin@gmail.com, ${ }^{2}$ Department of Physics, National University of Singapore, \\ ${ }^{3}$ Bioengineering Sciences Research Group, Faculty of Engineering and Environment, \\ University of Southampton, ${ }^{4}$ Corresponding Author. Currently at NEUROMATHCOMP, \\ Inria Sophia Antipolis - Méditerranée 2004, route des Lucioles - BP 9306902 Sophia \\ Antipolis Cedex, FRANCE.Tel.: +33(0)4923878 30, email james.maclaurin@inria.fr
}

\begin{abstract}
Fine sinusoidal wrinkling on the surfaces of mechanically compressed objects has been observed in many contexts over many years. In this paper we investigate such wrinkling through the application of a boundary layer analysis to an elastostatic problem in nonlinear elasticity. We determine the onset of buckling using a linear-stability analysis, and the leading-order postbuckling behaviour through consideration of higher-order terms of the energy. The object is assumed to (initially) 'preserve' its shape, so that these equations reduce to ordinary differential equations. We then apply a boundary-layer analysis to this problem, determining (in the asymptotic limit of large wavenumbers) the leading order behaviours of the eigenmode, the critical parameter, and the magnitude of the buckle. We find that the magnitude of a buckle with wavenumbers $\varsigma \gamma_{2}^{*}$ and $\varsigma \gamma_{3}^{*}$ (for fixed $\gamma_{2}^{*}$ and $\gamma_{3}^{*}$ ) has leading asymptotic order $\varsigma^{-\frac{3}{2}} \sqrt{\lambda_{(2)}}$, for an increment $\lambda_{(2)}$ of the critical parameter beyond the critical time of buckling. We provide electronic supplementary material which extends this analysis to that of incompressible elasticity. Finally we confirm the accuracy of our ansatz on a compressed NeoHookean ring.
\end{abstract}

\section{Introduction}

Wrinkling on the surface of nonlinearly elastic materials has been observed in many contexts over many years. The wrinkling is often observed in biological materials, for example on the surfaces of cells, tumours (Dervaux and Amar [8]), tubular organs (such as airways Moulton and Goriely [22], the oesophagus Li et al. [16], intestine and blood vessels MacLaurin et al. [19]) or brain convolutions. It is also observed in nonbiological materials, such as the wrinkling of stretched elastic sheets with low bending modulus (Brau et al. [2]), the wrinkling of thin films anchored to stiff substrates (Cao and Hutchinson [5]), the formation of sulci in compressed soft materials (Hohlfeld and Mahadevan [14]) or the wrinkling on the inner surface of a bent rubber block (Destrade et al. $[9])$.

These wrinkled patterns all occur in elastic materials in response to a gradual loading $\lambda$, whether by external forcing or internal growth fields, with the following characteristics. For small loading the unwrinkled initial state remains me- 
chanically stable. If wrinkling (buckling) does occur with wavenumbers $(n, \omega)$, there must be a critical value $\lambda^{(n, \omega)}$ of the loading such that the unwrinkled state is unstable to deformations of this type for loadings above $\lambda^{(n, \omega)}$ and stable below. This critical value $\lambda^{(n, \omega)}$ is found by superimposing an infinitesimally small sinusoidal deformation with these wavenumbers on the unwrinkled state, and searching for the value of $\lambda$ for which the wrinkled state has the same energy as the unwrinkled state. This only characterizes the solution at the critical point. To find the solution for values of $\lambda$ in the neighbourhood of the critical value, we need to analyze the higher order terms in the asymptotic expansion of the energy. The equations governing the post-buckled state are often difficult to analyze, but progress can be made if we make simplifying assumptions about the nature of the geometry and the solution.

We analyze the equations in the limit that the wavenumbers $(n, \omega)$ are large (and hence the wavelength is short), in which case the deformation is largely confined to a boundary layer at the surface. ${ }^{1}$ This assumption allows us to obtain a simplified expression for the shape of the buckled configuration in terms of geometric and material parameters. We assume that, if $(n, \omega)$ are the wavenumbers associated with a wrinkled pattern and $\lambda^{(n, \omega)}$ is the value of the critical parameter at which the wrinkled pattern first emerges, then $\lambda^{(n, \omega)}$ asymptotes to a finite constant as $(n, \omega) \rightarrow \infty$ (we define these variables more precisely below). In other words, we assume that the wrinkled patterns 'cluster together' as the wavelength shrinks. Furthermore we assume that the wrinkles are increasingly confined to the surface as $(n, \omega) \rightarrow \infty$. This phenomenon has been observed in previous studies of buckling in nonlinear elasticity (Biot [1], Coman and Bassom [7], Dervaux and Amar [8], Hohlfeld and Mahadevan [14], Cao and Hutchinson [5], MacLaurin et al. [19]). It is analogous to the surface buckling of compressed infinite half-spaces: since there is no characteristic lengthscale, all of the wavelengths go unstable simultaneously (Biot [1]). It has been studied in various contexts using boundary layer techniques (see particularly the papers by Coman [6] and Coman and Bassom [7]). A mathematical analysis using the theory of partial differential equations has also been performed by NegronMarrero and Montes-Pizarro [23, 24]. These authors conjecture a necessary and sufficient condition - the 'Complementing Condition' - for the clustering of the wavenumbers in the above manner, and they use this to investigate the nature of the buckling.

This paper has four main parts. In the first part (Section 2)- we outline an elastostatic problem and our assumptions on the pre-buckling behaviour of the system. In the second part (Sections $3-4$ ), we apply a linear stability analysis to determine when the system starts to buckle, before applying a postbuckling analysis to determine the leading order of the magnitude of the buckle. In the third part (Sections $5-6$ ), we perform a boundary layer analysis to elucidate the behaviour of the buckle for vanishingly small wavelengths. We will find that the magnitude of the buckle is of the order of the square root of the increment in the bifurcation parameter multiplied by $\varsigma^{-3 / 2}$ (where $\varsigma$ is of the order of the wavenumber). In the fourth part (Section 8) we apply the theory to the wrinkling of a compressed NeoHookean ring, and correlate the predictions of our boundary layer analysis with numerical simulations. We also provide ex-

\footnotetext{
${ }^{1}$ Our model is thus not accurate if there is a thin film on the surface.
} 
tra supplementary materials in which we extend our analysis to incompressible elasticity.

\section{Elastostatic Problem}

Our problem is that of a nonlinear elastic body subject to surface forces. We assume that there is no body force, although this paper could easily be extended to such a case. We let the reference configuration of the body be $\mathcal{B}_{0}$, with current configuration $\mathcal{B}$. Let $\left(X^{1}, X^{2}, X^{3}\right)$ be coordinates for $\mathcal{B}_{0}$ and $\left(x^{1}, x^{2}, x^{3}\right)$ be coordinates for $\mathcal{B}$. These coordinate systems are in $C^{\infty}$ correspondence with, respectively, $\mathcal{B}_{0}$ and $\mathcal{B}$. Throughout this paper, we denote coordinates in the current configuration with lower case letters and coordinates corresponding to the reference configuration with upper case letters. One can refer to [20] for a more detailed explanation of the differential geometry in this paper.

We assume that $\mathcal{B}_{0}$ and $\mathcal{B}$ are respectively parameterised in the following way

$$
\begin{aligned}
& X^{1} \in\left[X_{c}^{1}, X_{+}^{1}\right], X^{2} \in\left[-X_{c}^{2}, X_{c}^{2}\right], X^{3} \in\left[-X_{c}^{3}, X_{c}^{3}\right] . \\
& x^{1} \in\left[x_{c}^{1}, x_{+}^{1}\right], x^{2} \in\left[-x_{c}^{2}, x_{c}^{2}\right], x^{3} \in\left[-x_{c}^{3}, x_{c}^{3}\right] .
\end{aligned}
$$

We assume throughout this paper that $\left\{x_{c}^{1}, x_{c}^{2}, x_{c}^{3}\right\}$ are finite, although the analysis may easily be extended to the cases that some or all of $\left\{x_{+}^{1}, x_{c}^{2}, x_{c}^{3}\right\}$ are infinite. Let the covariant basis vectors for $\mathcal{B}$ be $\left\{\mathbf{g}_{a}\right\}$ and the covariant basis vectors for $\mathcal{B}_{0}$ be $\left\{\mathbf{G}_{A}\right\}$. The corresponding metrics are $g_{a b}=\mathbf{g}_{a} \cdot \mathbf{g}_{b}$ and $G_{A B}=\mathbf{G}_{A} \cdot \mathbf{G}_{B}$. Let $\mathbf{u}=\mathbf{x}-\mathbf{X}$ be the displacement. We consider $\mathbf{u}=u^{a} \mathbf{g}_{a}$ to be a vector in current coordinates (one may 'shift' $\mathbf{X}$ to the current coordinates in the manner of Marsden and Hughes [20] in order that this is well-defined). Let $\partial \mathcal{B}^{(a)}$ be the subset of $\partial \mathcal{B}$ given by $x^{a}=$ constant. We assume that the normal to $\partial \mathcal{B}^{(a)}$ is given by either $\mathbf{g}_{a}$ or $-\mathbf{g}_{a}$. We assume that the normal to $\partial \mathcal{B}^{(a)}$ in reference coordinates is given by $\mathbf{G}_{A}$ (or its negative), so that (in reference coordinates) the boundary is $X^{a}=$ constant. Of course, $\cup_{a} \partial \mathcal{B}^{(a)}=\partial \mathcal{B}$.

We assume the bifurcation is driven by a single parameter $\lambda$, which is initially equal to zero before being gradually increased until bifurcation occurs. We denote the state the system initially adopts the base state. We assume that initially, when $\lambda=0$, the system is mechanically stable. As $\lambda$ increases, we assume that a boundary layer (with progressively finer wrinkling) develops on the face $\partial \mathcal{B}^{*}$ given by $x^{1}=x_{c}^{1}$. We will perform a stability analysis in Sections 3 and 4 to determine when the system destabilises, and what is the postbuckling behaviour. We will then apply a boundary layer analysis to this phenomenon in Section 5 so that we may elucidate its behaviour. However before we do all this we must firstly outline the equations of elastostatic equilibrium in greater depth.

We suppose that the deformation is given by $\phi$, i.e. $\mathbf{x}=\phi(\mathbf{X}, \lambda)$. Let $\mathbf{F}$ be the deformation gradient, with components $F^{a}{ }_{A}=\frac{\partial \phi^{a}}{\partial X^{A}}$. Let $J$ be the Jacobian of $\phi$, i.e.

$$
J=\operatorname{det} \mathbf{F}
$$

where det is the standard matrix determinant. This gives the local volumetric 'stretch' of the material under the deformation $\phi$. We require a similar expression for the local stretch in area at each of the boundaries. We begin by 
obtaining such an expression at the boundary $\partial \mathcal{B}^{(1)}$. Because $x^{1}$ is constant over this boundary, the area of a small segment of it (spanned by small increments $d x^{2}$ and $d x^{3}$ in current coordinates) is given by $d a=\Gamma d x^{2} d x^{3}$, where

$$
\Gamma=\left|\left(\mathbf{g}_{2} \times \mathbf{g}_{3}\right) \cdot \mathbf{n}\right|
$$

and $\mathbf{n}=\left(g^{11}\right)^{-\frac{1}{2}} \mathbf{g}^{1}$ is the unit outer normal. The same segment has area (in reference coordinates) given by $d A=\Gamma_{0} d X^{2} d X^{3}$, where

$$
\Gamma_{0}=\left|\left(\mathbf{G}_{2} \times \mathbf{G}_{3}\right) \cdot \mathbf{N}\right|
$$

and $\mathbf{N}=\left(G^{11}\right)^{-\frac{1}{2}} \mathbf{G}^{1}$ is the unit outer normal. We define $\Gamma$ and $\Gamma_{0}$ over $\partial \mathcal{B}^{(2)}$ and $\partial \mathcal{B}^{(3)}$ analogously.

We assume that the body is hyperelastic, so that there exists a strain energy function $\psi(\mathbf{F}, \mathbf{X}, \lambda)$, which gives the stored elastic energy per unit volume of $\mathcal{B}_{0}$. The first Piola-Kirchhoff stress tensor $\mathbf{P}_{0}$ has components $P_{0}^{a A}=g^{b a} \frac{\partial \psi}{\partial F^{b} A}$. The system is in mechanical equilibrium, so that over $\mathcal{B}_{0}$,

$$
-\nabla_{0} \cdot \mathbf{P}_{0}=\mathbf{0}
$$

where $\nabla_{0}$ is the divergence over reference coordinates and we assume there is no body force. For the boundary conditions, we assume that $\partial \mathcal{B}_{0}$ can be decomposed as $\partial \mathcal{B}_{0}=\partial \mathcal{B}_{0}^{u} \cup \partial \mathcal{B}_{0}^{t}$, where $\partial \mathcal{B}_{0}^{u}$ and $\partial \mathcal{B}_{0}^{t}$ are distinct closed sets. We assume the same decomposition applies for $\partial \mathcal{B}$. On $\partial \mathcal{B}_{0}^{t}$, we impose the traction boundary condition

$$
\mathbf{P}_{0} \cdot \mathbf{N}=\mathbf{t}(\mathbf{X}, \mathbf{u}, \lambda)
$$

where $\mathbf{t}$ gives the surface traction per unit area of $\partial \mathcal{B}_{0}^{t}$ and $\mathbf{N}$ is the unit outer normal. On $\partial \mathcal{B}_{0}^{u}$ we impose the displacement boundary condition

$$
\mathbf{u}=\mathbf{v}(\mathbf{X}, \lambda),
$$

where $\mathbf{v}$ is the stipulated displacement function.

We assume for simplicity throughout this document that $\phi$ is infinitely continuous and differentiable with respect to both $\nabla(X)$ and $\lambda$ (although of course this assumption could be significantly relaxed without loss of the major results). We similarly assume that $\mathbf{v}$ (and $\mathbf{t}$ ) are infinitely continuously differentiable with respect to $\lambda$ (and $\mathbf{u}$ ).

We apply the Piola Transform to find that, over current coordinates, $P^{a b}=$ $J^{-1} P_{0}^{a B} F^{b}{ }_{B}$. Here and after, unless otherwise indicated, every tensor is over current coordinates. The equation of equilibrium (4) thus becomes

$$
-\nabla \cdot \mathbf{P}=\mathbf{0},
$$

where the divergence is taken over current coordinates. The stress boundary condition (5) may similarly be written as

$$
\mathbf{P} \cdot \mathbf{n}=\frac{\Gamma_{0}}{\Gamma} \mathbf{t}(\mathbf{X}, \mathbf{F}, \lambda)
$$

where we have scaled by $\Gamma_{0} / \Gamma$ to account for the change in area induced by the 
deformation.

We require $\mathbf{t}$ to have a potential $\tau(X, \mathbf{u}, \lambda)$ per unit area of $\partial \mathcal{B}_{0}^{t}$, such that the first variation may be written as

$$
\frac{\partial \tau}{\partial u_{a}} w_{a}=-w_{c} t^{c} .
$$

For example, we may write the potential of a dead load $\mathbf{h}(\mathbf{X}, \lambda)$ applied to a face $\mathcal{B}^{t}$ as

$$
\tau=\mathbf{u} \cdot \mathbf{h} .
$$

In summary, the total potential of the system is given by

$$
W=\iiint_{\mathcal{B}_{0}} \psi d V_{0}+\iint_{\mathcal{B}_{0}^{t}} \tau d A_{0},
$$

where $d V_{0}=\operatorname{det} G_{A B} d X^{1} d X^{2} d X^{3}$ and $d A_{0}=\Gamma_{0} d X^{J} d X^{K}$ over $\partial \mathcal{B}^{(L)}$ (where $\{J, K, L\}=\{1,2,3\})$. Over current coordinates, we find

$$
W=\iiint_{\mathcal{B}} J^{-1} \psi d V+\iint_{\mathcal{B}^{t}} \frac{\Gamma_{0}}{\Gamma} \tau d A,
$$

where $d V=\operatorname{det} g_{a b} d x^{1} d x^{2} d x^{3}$ and $d A=\Gamma d x^{j} d x^{k}$ over $\partial \mathcal{B}^{(l)}$ (where $\{j, k, l\}=$ $\{1,2,3\})$.

\subsection{Incremental Notation}

Here we outline expressions governing the incremental behaviour of the stress tensor and the surface forces: refer to Ogden [26] for a more detailed explanation. These are essentially obtained by differentiating the respective quantities with respect to the displacement (and its gradient). Before we begin, we recall that the dot product between two vectors is

$$
\mathbf{v} \cdot \mathbf{w}=v^{a} w_{a}=v_{a} w^{a} .
$$

We illustrate the double contraction between two tensors with the following two examples. Let $\mathbf{T}$ be $T^{a}{ }_{B}, \mathbf{S}$ be $S^{c}{ }_{D}{ }^{e} F, \mathbf{Y}$ be $Y_{g}{ }^{H}$ and $\mathbf{Z}$ be $Z_{i}{ }^{J}$. Then $T: Y=T_{a}{ }^{B} Y^{a}{ }_{B}$ and $S: Y: Z=S^{a}{ }_{B}{ }^{c}{ }_{D} Y_{a}{ }^{B} Z_{c}{ }^{D}$. The double contraction of higher order tensors may be defined analogously.

The fixed-reference elastic moduli (in the terminology of Ogden [26]) are

$$
\begin{aligned}
& P_{0}^{a B}{ }_{c}{ }^{D}=\frac{\partial P_{0}^{a B}}{\partial F^{c} D}, \\
& P_{0}^{a B}{ }_{c}{ }^{D} e^{F}=\frac{\partial^{2} P_{0}^{a B}}{\partial F^{c}{ }_{D} \partial F^{e}{ }_{F}}, \\
& P_{0}^{a B}{ }_{c}{ }^{D}{ }^{F}{ }_{g}{ }^{H}=\frac{\partial^{3} P_{0}^{a B}}{\partial F^{c}{ }_{D} \partial F^{e}{ }_{F} \partial F^{g} H} \text {. }
\end{aligned}
$$

As we will be working in the current configuration, we must push the fixedreference moduli forward to the current configuration, obtaining the instanta- 
neous elastic moduli, i.e.

$$
\begin{aligned}
P^{a b}{ }_{c}^{d} & =J^{-1} P_{0}^{a L}{ }_{c}{ }^{K} F^{d}{ }_{K} F^{b}{ }_{L}, \\
P^{a b{ }_{c}{ }^{d} e^{f}} & =J^{-1} P_{0}^{a L}{ }_{c}{ }^{K}{ }_{e}{ }^{J} F^{f}{ }_{J} F^{d}{ }_{K} F^{b}{ }_{L}, \\
P^{a b}{ }_{c}{ }^{d} e^{f}{ }_{g}{ }^{h} & =J^{-1} P_{0}^{a L}{ }_{c}{ }^{K}{ }^{J}{ }^{J}{ }_{g}{ }^{M} F^{f}{ }_{J} F^{d}{ }_{K} F^{b}{ }_{L} F^{h}{ }_{M} .
\end{aligned}
$$

Let $\mathbf{u}$ be a vector field over $\mathcal{B}$. Its covariant derivative is

$$
\nabla \mathbf{u}=u_{\mid b}^{a} \mathbf{g}_{a} \otimes \mathbf{g}^{b},
$$

where $u^{a}{ }_{\mid b}=\frac{\partial u^{a}}{\partial x^{b}}+\Gamma_{c b}^{a} u^{c}$, and $\left\{\Gamma_{c b}^{a}\right\}$ are the Christoffel symbols. We note that $u_{a \mid b}=u^{c}{ }_{\mid b} g_{c a}$. Thus if $\mathbf{s}, \mathbf{u}, \mathbf{w}, \mathbf{z}$ are vector fields over $\mathcal{B}$, we may write the increments in the energy in the form

$$
\begin{aligned}
& \frac{\partial^{2} \psi}{\partial F^{2}}: \nabla \mathbf{w}: \nabla \mathbf{z}=P^{a b}{ }_{c}^{d} w_{a \mid b} z^{c}{ }_{\mid d}, \\
& \frac{\partial^{3} \psi}{\partial F^{3}}: \nabla \mathbf{u}: \nabla \mathbf{w}: \nabla \mathbf{z}=P^{a b}{ }_{c}{ }^{d} e^{f} u_{a \mid b} w^{c}{ }_{\mid d} z^{e}{ }_{\mid f}, \\
& \frac{\partial^{4} \psi}{\partial F^{4}}: \nabla \mathbf{s}: \nabla \mathbf{u}: \nabla \mathbf{w}: \nabla \mathbf{z}=P^{a b}{ }_{c}^{d} e^{f}{ }_{g}{ }^{h} s_{a \mid b} u_{\mid d}^{c} w^{e}{ }_{\mid f} z^{g}{ }_{\mid h} .
\end{aligned}
$$

We write $\frac{D^{j} \tau}{D \mathbf{u}^{j}}$ to be the order $j$ contravariant vector such that

$$
\left(\frac{D^{j} \tau}{D \mathbf{u}^{j}}\right)_{a_{1} \ldots a_{j}}=\frac{\Gamma_{0}}{\Gamma} \frac{d^{j} \tau}{d u^{a_{1}} \ldots d u^{a_{j}}} .
$$

Similarly $\frac{D^{j} \mathbf{t}}{D \mathbf{u}^{j}}=-\frac{D^{j+1} \tau}{D \mathbf{u}^{j+1}}$.

The divergence of a second order contravariant tensor $\mathbf{P}$ defined over the current configuration is

$$
(\nabla \cdot \mathbf{P})^{a}=\frac{\partial}{\partial x^{b}} P^{a b}+P^{k b} \Gamma_{k b}^{a}+P^{a k} \Gamma_{k b}^{b} .
$$

\section{Stability Analysis}

There are two basic components of our stability analysis. In the first step, we employ a linear stability analysis to determine the value(s) of $\lambda$ for which the system bifurcates. In the next step, we perform a perturbation expansion about the point of bifurcation to determine the leading order of the magnitude of the buckle and its stability.

We work in the current configuration $\mathcal{B}$. We recall that the base state is the configuration prior to buckling, with displacement $\mathbf{u}_{(0)}$ and $\lambda=\lambda_{(0)}$. We insist that the base state deformation 'preserves' the geometry in the following sense. The base state solution is obtained by solving (4)-(6) for $\mathbf{u}$ (for the base state solution we consider $\mathbf{u}$ to be a vector over $\mathcal{B}_{0}$, so that its coordinates are with respect to $\left\{G_{a}\right\}$ ). We assume that the strain energy function $\psi$, the potential $\tau$, boundary displacement $\mathbf{v}$ and the geometry variables $\left\{\Gamma_{0}, G_{A B}\right\}$ may be written as functions of $X^{1}, u_{(0)}^{1}$ and $\lambda$ only (so that they are independent of $\left.\left\{u_{(0)}^{2}, u_{(0)}^{3}, X^{2}, X^{3}\right\}\right)$. We assume that the base state solution of (4)-(6) is such 
that $x^{2}=X^{2}, x^{3}=X^{3}$, that $\mathbf{g}_{\alpha}$ and $\mathbf{G}_{\alpha}$ are coaxial (for $\alpha \in\{1,2,3\}$ ) and $\mathbf{u}_{0}$ may be written as a function of $X^{1}$ only $^{2}$. Furthermore, we assume that the solution $\mathbf{u}_{0}$ is infinitely continuous and differentiable with respect to $X^{1}$ and $\lambda$ that the perturbation analysis to follow is well-defined. Note that $u_{(0)}^{j}$ may be nonzero (for $j=2$ or 3 ). Most of the phenomena outlined in the introduction with either a rectangular or cylindrical geometry satisfy these assumptions ${ }^{3}$. We note also that many of the above assumptions may be relaxed without affecting the major results in this paper: it depends on the specific nature of the problem.

We consider the stability of the base state to be governed by the incremental equations of equilibrium (Ogden [26]). This requires us to consider the effect of a small variation $\epsilon \mathbf{u}_{(1)}$ on the stability of the system, so that the total displacement is of the form

$$
\mathbf{u}=\mathbf{u}_{(0)}+\epsilon \mathbf{u}_{(1)} .
$$

The displacement increment $\mathbf{u}_{(1)}$ must be admissible, meaning that it is in $L^{2}(\mathcal{B})$ and satisfies the 'incremental boundary condition'

$$
\mathbf{u}_{(1)}=\mathbf{0} \text { over } \partial \mathcal{B}^{u}
$$

We denote the set of all admissible displacements by $\mathcal{U}$.

We denote the state with displacement field (13), State I. Let $\triangle W=\epsilon W_{(1)}+$ $\frac{1}{2} \epsilon^{2} W_{(2)}+\ldots$ be the difference in energy between the base state and State I. We find that to leading order

$$
\begin{aligned}
W_{(1)} & =\iiint_{\mathcal{B}} \frac{\partial \psi}{\partial \mathbf{F}}: \nabla \mathbf{u}_{(1)} d V+\iint_{\partial \mathcal{B}^{t}} \frac{D \tau}{D \mathbf{u}} \cdot \mathbf{u}_{(1)} d A \\
W_{(2)} & =\iiint_{\mathcal{B}} \frac{\partial^{2} \psi}{\partial \mathbf{F}^{2}}: \nabla \mathbf{u}_{(1)}: \nabla \mathbf{u}_{(1)} d V \\
& +\iint_{\partial \mathcal{B}^{t}} \frac{D^{2} \tau}{D \mathbf{u}^{2}} \cdot \mathbf{u}_{(1)} \cdot \mathbf{u}_{(1)} d A
\end{aligned}
$$

Here, and after, $d A$ and $d V$ are the area and volume elements given in (11). Now $W_{(1)}$ is identically zero as a consequence of the base state being in mechanical equilibrium. The second of these terms $\left(W_{(2)}\right)$ governs the stability of the base state: the base state is stable if $(16)$ is greater than zero for all nonzero admissible $\mathbf{u}_{(1)}$, and it is unstable if (16) is less than zero. At a point of bifurcation, (16) must be zero for some $\mathbf{u}_{(1)}$. The Trefftz Criterion states that a bifurcation point occurs when the first variation of (16) (considered as a function of $\mathbf{u}_{(1)}$ ) is zero. That is, if we superpose a further admissible variation $\mathbf{w}_{(1)}$ on top of

\footnotetext{
${ }^{2}$ It follows by implication that $g_{a b}$ and $\Gamma_{b c}^{a}$ may be written as functions of $X^{1}, u_{(0)}^{1}$ only.

${ }^{3}$ The problems with a spherical geometry may be analysed using a similar method to this paper, except that one must use the spherical harmonics to perform the stability analysis, see Wang and Ertepinar [28].
} 
$\mathbf{u}_{(1)}$ and neglect terms of quadratic order,

$$
\begin{aligned}
\frac{D W_{(2)}}{D \mathbf{u}_{(1)}} \cdot \mathbf{w}_{(1)}=2 \iiint_{\mathcal{B}} \frac{\partial^{2} \psi}{\partial \mathbf{F}^{2}}: \nabla \mathbf{u}_{(1)} & : \nabla \mathbf{w}_{(1)} d V \\
& -2 \iint_{\partial \mathcal{B}^{t}} \frac{D \mathbf{t}}{D \mathbf{u}} \cdot \mathbf{u}_{(1)} \cdot \mathbf{w}_{(1)} d A=0
\end{aligned}
$$

for all admissible $\mathbf{w}_{(1)}$. The resulting Euler-Lagrange equations are

$$
\begin{gathered}
\mathbf{L}\left(\mathbf{u}_{(1)}\right)=\mathbf{0}, \text { over } \mathcal{B} \\
\mathbf{M}\left(\mathbf{u}_{(1)}\right)=\mathbf{0} \text { over } \partial \mathcal{B}^{t}
\end{gathered}
$$

where

$$
\begin{aligned}
\mathbf{L}(\mathbf{w}) & =\nabla \cdot \mathbf{P}_{(1)}, \\
M(\mathbf{w})^{a} & =P_{(1)}{ }^{a b}{ }_{c}^{d} w^{c}{ }_{\mid d} n_{b}-\frac{\Gamma_{0}}{\Gamma} \frac{\partial t^{a}}{\partial u^{c}} w^{c}, \\
P_{(1)}{ }^{a b} & =P^{a b}{ }_{c}^{d} w^{c}{ }_{\mid d},
\end{aligned}
$$

and $\mathbf{n}$ is the unit outer normal over $\mathcal{B}^{t}$. We determine a point of bifurcation by gradually incrementing $\lambda_{(0)}$ until there exists a nonzero solution to (14),(18) and (19).

\subsection{Fourier Decomposition}

We may investigate the stability of the various modes by assuming $\mathbf{u}_{(1)}$ to be of the following form,

$$
\begin{aligned}
\mathbf{u}_{(1)}=a_{+,+}^{\left(\gamma_{2}, \gamma_{3}\right)} \mathbf{u}_{(1)}^{\left(\gamma_{2}, \gamma_{3}\right)}+a_{-,-}^{\left(\gamma_{2}, \gamma_{3}\right)} & \mathbf{u}_{(1)}^{\left(-\gamma_{2},-\gamma_{3}\right)} \\
& +a_{-,+}^{\left(\gamma_{2}, \gamma_{3}\right)} \mathbf{u}_{(1)}^{\left(-\gamma_{2}, \gamma_{3}\right)}+a_{+,-}^{\left(\gamma_{2},-\gamma_{3}\right)} \mathbf{u}_{(1)}^{\left(\gamma_{2},-\gamma_{3}\right)},
\end{aligned}
$$

where $\left\{a_{ \pm, \pm}^{\left(\gamma_{2}, \gamma_{3}\right)}\right\}$ are constants and

$$
u_{(1)}^{(\alpha, \beta)}:=\mathbf{Z}^{(\alpha, \beta)} e^{i \alpha x^{2}} e^{i \beta x^{3}}
$$

for some function $\mathbf{Z}^{(\alpha, \beta)}\left(x^{1}, \lambda\right)$. We assume that $\gamma_{2}=\frac{m \pi}{2 x_{2}^{c}}$ for some $m \in \mathbb{Z}$ and similarly for $\gamma_{3}$ (recall that we are also assuming that $x_{2}=X_{2}$ and $x_{3}=X_{3}$ ). Let $\mathcal{U}_{\alpha, \beta}$ be the set of all displacements of the form (24).

It follows from our earlier assumptions that (14), (18), and (19) reduce to an ordinary differential equation in $x^{1}$. In order that this ordinary differential equation is solveable, we require that $\mathbf{L}$ is strongly elliptic, meaning that (for a fixed base state) there exists a positive $K$ such that for all $a$,

$$
\frac{\partial P^{a}{ }_{1}}{\partial F^{a}{ }_{1}}=P^{a}{ }_{1 a}^{1}>K \text { over } \mathcal{B} \text {. }
$$

In this definition of strong ellipticity we are considering $\mathbf{L}$ as an ordinary differential operator in $x^{1}$ due to the substitution in (24). Our definition differs from the conventional definition of strong ellipticity in nonlinear elasticity, where $\mathbf{L}$ is 
considered as a partial differential operator [20]. Since (by our earlier assumptions on $\psi$ and $\tau$ ), the coefficients of $\mathbf{L}$ and $\mathbf{M}$ are infinitely continuous and differentiable, the solution of the form (24) is unique and infinitely continuous and differentiable (although possibly zero) (Evans [10]). Furthermore, it follows from the self-adjointness of $\mathbf{L}$, the Fourier decomposition and (25) that

$$
L^{2}(\mathcal{B})=\operatorname{Range} \mathbf{L} \oplus \operatorname{Ker} \mathbf{L} .
$$

This is known as the Fredholm Alternative. The Fredholm Alternative is necessary for the postbuckling analysis to be performed in the following section. Let $\mathcal{U}_{(\alpha, \beta)}$ be the set of all admissible $\mathbf{u}$ which can be written in the form (24), and let $\lambda^{\left(\gamma_{2}, \gamma_{3}\right)}$ be the value of $\lambda$ for which a solution of the form (24) exists to (14), (18) and (19). We require that $\mathbf{u}_{(1)}^{\left(\gamma_{2}, \gamma_{3}\right)} \neq \mathbf{0}$.

It is necessary that each of $\left\{\mathbf{u}_{(1)}^{\left( \pm \gamma_{2}, \pm \gamma_{3}\right)}\right\}$ must individually satisfy (18). It can be seen that, as a consequence of taking the complex conjugate, we may write

$$
\begin{aligned}
\overline{\mathbf{u}}_{(1)}^{\left(\gamma_{2}, \gamma_{3}\right)} & =\mathbf{u}_{(1)}^{\left(-\gamma_{2},-\gamma_{3}\right)} \text { and } \\
\lambda^{\left(-\gamma_{2},-\gamma_{3}\right)} & =\lambda^{\left(\gamma_{2}, \gamma_{3}\right)} .
\end{aligned}
$$

If

$$
\lambda^{\left(-\gamma_{2}, \gamma_{3}\right)} \neq \lambda^{\left(\gamma_{2}, \gamma_{3}\right)}
$$

then we must insist that $\mathbf{u}_{(1)}^{\left(-\gamma_{2}, \gamma_{3}\right)}=\mathbf{u}_{(1)}^{\left(\gamma_{2},-\gamma_{3}\right)}=\mathbf{0}$. Aside from these cases, we assume that the modes are distinct, i.e. $\lambda^{\left(\beta_{1}, \beta_{2}\right)} \neq \lambda^{\left(\delta_{1}, \delta_{2}\right)}$ if $\beta_{1} \neq \pm \delta_{1}$ or $\beta_{2} \neq \pm \delta_{2}$.

\section{Postbuckling Analysis}

In this section we perform a perturbation expansion at the critical time of buckling of a mode $\left(\gamma_{2}, \gamma_{3}\right)$. Thus the expansion is at $\lambda=\lambda^{\left(\gamma_{2}, \gamma_{3}\right)}$, and there exists a solution of the form (23) to (14), (18) and (19). Our method is to obtain the displacement field by successively rendering each order of $\triangle W$ stationary. We are essentially employing the energy criterion of Lagrange: we are assuming that the system adopts the state which minimises its potential energy. We may stipulate that $\mathbf{u}_{(k)} \perp \mathbf{u}_{(1)}$ (for $k \geq 2$ ), so that $\epsilon$ modulates the magnitude of the buckle in the direction of the eigenmode. This stipulation is possible because of the Fredholm Alternative (26). We may then determine $\mathbf{u}_{(k)}$ to be the unique displacement field which renders the $O\left(\epsilon^{2 k}\right)$ component of the energy stationary. Our method is an adaptation of that outlined in Koiter [15] to three-dimensional elasticity. It has recently been employed to study the emergence of wrinkling in growing Blatz-Ko materials by the authors [19] and compressed Neo-Hookean materials by Cao and Hutchinson [4].

The increment in $\lambda$ is assumed to be of higher order than the increment in the displacement, i.e.

$$
\lambda=\lambda_{(0)}+\frac{1}{2} \epsilon^{2} \lambda_{(2)}+\ldots,
$$

where $\lambda_{(0)}=\lambda^{\left(\gamma_{2}, \gamma_{3}\right)}$. This is necessary for the higher order terms in the perturbation expansion to be well-matched. We expand the change in energy from 
the buckled to unbuckled state as

$$
\Delta W=\epsilon W_{(1)}+\frac{1}{2} \epsilon^{2} W_{(2)}+\frac{1}{6} \epsilon^{3} W_{(3)}+\frac{1}{24} \epsilon^{4} W_{(4)}+\ldots
$$

We define

$$
\begin{aligned}
& \bar{W}(\mathbf{w})=\iiint_{\mathcal{B}} \frac{\partial \psi}{\partial \mathbf{F}}: \nabla \mathbf{w} d V+\iint_{\partial \mathcal{B}^{t}} \frac{D \tau}{D \mathbf{u}} \cdot \mathbf{w} d A \\
& \tilde{W}(\mathbf{w})=\iiint_{\mathcal{B}} \frac{\partial^{2} \psi}{\partial \mathbf{F}^{2}}: \nabla \mathbf{u}_{(1)}: \nabla \mathbf{w} d V+\iint_{\partial \mathcal{B}^{t}} \frac{D^{2} \tau}{D \mathbf{u}^{2}} \cdot \mathbf{u}_{(1)} \cdot \mathbf{w} d A .
\end{aligned}
$$

In fact both of these expressions are identically zero for all admissible w. In the case of $\bar{W}(\mathbf{w})$, this is a consequence of the base state being in mechanical equilibrium. In the case of $\tilde{W}$, this is a consequence of the Trefftz condition (17). We may thus expand the subsequent orders of the energy as follows,

$$
\begin{gathered}
W_{(1)}=\bar{W}\left(\mathbf{u}_{(1)}\right) \\
\frac{1}{2} W_{(2)}=\frac{1}{2} \bar{W}\left(\mathbf{u}_{(2)}\right)+\frac{1}{2} \tilde{W}\left(\mathbf{u}_{(1)}\right) . \\
\frac{1}{6} W_{(3)}=\frac{1}{6} \bar{W} \mathbf{u}_{(3)}+\frac{1}{2} \frac{\partial}{\partial \lambda} \bar{W}\left(\mathbf{u}_{1}\right) \lambda_{(2)}+\frac{1}{2} \tilde{W}\left(\mathbf{u}_{(2)}\right) \\
+\iiint_{\mathcal{B}} \frac{1}{6} \frac{\partial^{3} \psi}{\partial \mathbf{F}^{3}}: \nabla \mathbf{u}_{(1)}: \nabla \mathbf{u}_{(1)}: \nabla \mathbf{u}_{(1)} d V \\
+\iint_{\partial \mathcal{B}^{t}} \frac{1}{6} \frac{D^{3} \tau}{D \mathbf{u}^{3}} \cdot \mathbf{u}_{(1)} \cdot \mathbf{u}_{(1)} \cdot \mathbf{u}_{(1)} d A
\end{gathered}
$$

In the expression for $W_{(4)}$ below, we have neglected quadratic terms in $\lambda_{(2)}$ since they integrate to zero as a consequence of the regularity of the geometry.

$$
\begin{array}{r}
\frac{1}{24} W_{(4)}=\frac{1}{24} \bar{W}\left(\mathbf{u}_{(4)}\right)+\frac{1}{6} \tilde{W}\left(\mathbf{u}_{(3)}\right)+\frac{1}{4} \frac{\partial}{\partial \lambda}\left(\tilde{W}\left(\mathbf{u}_{1}\right)+\bar{W}\left(\mathbf{u}_{2)}\right)\right) \lambda_{(2)} \\
+\iiint_{\mathcal{B}}\left[\frac{1}{24} \frac{\partial^{4} \psi}{\partial \mathbf{F}^{4}}: \nabla \mathbf{u}_{(1)}: \nabla \mathbf{u}_{(1)}: \nabla \mathbf{u}_{(1)}: \nabla \mathbf{u}_{(1)}+\right. \\
\left.\frac{1}{4} \frac{\partial^{3} \psi}{\partial \mathbf{F}^{3}}: \nabla \mathbf{u}_{(2)}: \nabla \mathbf{u}_{(1)}: \nabla \mathbf{u}_{(1)}+\frac{1}{8} \frac{\partial^{2} \psi}{\partial \mathbf{F}^{2}}: \nabla \mathbf{u}_{(2)}: \nabla \mathbf{u}_{(2)}\right] d V \\
+\iint_{\partial \mathcal{B}^{t}}\left[\frac{1}{24} \frac{D^{4} \tau}{D \mathbf{u}^{4}} \cdot \mathbf{u}_{(1)} \cdot \mathbf{u}_{(1)} \cdot \mathbf{u}_{(1)} \cdot \mathbf{u}_{(1)}+\frac{1}{4} \frac{D^{3} \tau}{D \mathbf{u}^{3}} \cdot \mathbf{u}_{(2)} \cdot \mathbf{u}_{(1)} \cdot \mathbf{u}_{(1)}\right. \\
\left.+\frac{1}{8} \frac{D^{2} \tau}{D \mathbf{u}^{2}} \cdot \mathbf{u}_{(2)} \cdot \mathbf{u}_{(2)}\right] d A .
\end{array}
$$

We will determine each order of the displacement by successively rendering each order of the energy stationary. We have already seen that $W_{(1)}$ is zero as a consequence of the base state being in equilibrium. It follows from the Trefftz Criterion for buckling that $\mathbf{u}_{(1)}$ renders $W_{(2)}$ stationary. However the magnitude of $\mathbf{u}_{(1)}$ (i.e. the constants $\left\{a_{ \pm, \pm}^{\left(\gamma_{2}, \gamma_{3}\right)}\right\}$ ) remains undetermined: we determine this through consideration of the higher orders of the energy. 
We must make a small detour and discuss the nature of this stationary point of $W_{(2)}$. If $\left(\gamma_{2}, \gamma_{3}\right)$ is the first mode to go unstable, then (16) is positive semidefinite and this stationary point of $W_{(2)}$ will be a minimum. However if another mode $(\alpha, \beta)$ has already gone unstable before $\left(\gamma_{2}, \gamma_{3}\right)$ then we expect that $W_{(2)}<$ 0 if $\mathbf{u}_{(1)}=\mathbf{u}_{(1)}^{(\alpha, \beta)}$. This means that the mode $\left(\gamma_{2}, \gamma_{3}\right)$ branch is unstable to perturbations of this form (for $\epsilon$ small enough). One might expect the system to move outside the regime of accuracy of our perturbation expansion. However we are still interested in investigating the mode $\left(\gamma_{2}, \gamma_{3}\right)$ buckled state even if it is not the first to go unstable, for the following reasons. In some systems, the critical times $\left\{\lambda^{(\alpha, \beta)}\right\}$ of the modes are highly clustered. Indeed this clustering of the modes is precisely what we wish to investigate with our boundary layer analysis. Sometimes, for example, buckles of infinitely fine wavelength are the first to go unstable, so that for every set of modes $\left(\gamma_{2}, \gamma_{3}\right)$ there is another set of modes $\left(\gamma_{2}^{*}, \gamma_{3}^{*}\right)$ which goes unstable before (see for example MacLaurin et al. [19]). We expect that every continuum model becomes inaccurate as the wavelength asymptotes to zero. Thus, in practice, systems will not necessarily realise the first modes to go unstable (if this even exists). Furthermore there may be small inhomogeneities which predispose the system more towards buckles of one particular type (which are not the first to go unstable): if the modes are highly clustered then these inhomogeneities may be sufficient to reverse the buckling order of the various modes. In conclusion, we assume that it is possible that the system might adopt the mode $\left(\gamma_{2}, \gamma_{3}\right)$ buckled state even if $\mathbf{u}_{(1)}$ does not minimise $W_{(2)}$. Since $W_{2}$ is only stationary with respect to $\mathbf{u}_{(1)}$, we must investigate $W_{3}$ and $W_{4}$ to further understand the nature of the bifurcation.

It follows from our assumptions about the geometry and base state deformation that $W_{(3)}$ is identically zero. In more detail, the first two terms of (35) are zero due to (31)-(32). The incremental elastic moduli are functions of $x^{1}$ and $\lambda$ only due to our assumptions about the base state deformation outlined in Section 3. Thus the other terms integrate to zero due to the regularity of our geometry. It follows that we must determine the magnitude of the buckle using $W_{(4)}$. We employ the Liapunov-Schmidt reduction to do this. In brief, we do this by fixing $\mathbf{u}_{(1)}$ and $\lambda_{(2)}$ and rendering the terms containing $\mathbf{u}_{(2)}$ stationary. Upon doing this we obtain an expression for $\mathbf{u}_{(2)}$ as a function of $\left\{a_{ \pm, \pm}^{\left(\gamma_{2}, \gamma_{3}\right)}\right\}$ and $\lambda_{(2)}$. We may then, in turn, determine $\left\{a_{ \pm, \pm}^{\left(\gamma_{2}, \gamma_{3}\right)}\right\}$ by requiring them to render the resulting expression for the energy stationary.

\subsection{Solution of $\mathbf{u}_{(2)}$}

We begin by specifying that $\mathbf{u}_{(2)}$ renders the terms in (36) stationary to admissible variations, with fixed $\mathbf{u}_{(1)}$ and $\lambda_{(2)}$. We decompose $\mathbf{u}_{2}$ such that $\mathbf{u}_{2}=\mathbf{u}_{(2, a)}+\lambda_{(2)}^{\left(\gamma_{2}, \gamma_{3}\right)} \mathbf{u}_{(2, p)}$, where for all admissible variations $\mathbf{w}$ (we defined admissibility in Section 3 ),

$$
\begin{aligned}
\iiint_{\mathcal{B}}\left[\frac{\partial^{2} \psi}{\partial \mathbf{F}^{2}}\right. & \left.: \nabla \mathbf{u}_{(2, a)}: \nabla \mathbf{w}+\frac{\partial^{3} \psi}{\partial \mathbf{F}^{3}}: \nabla \mathbf{u}_{(1)}: \nabla \mathbf{u}_{(1)}: \nabla \mathbf{w}\right] d V \\
& +\iint_{\partial \mathcal{B}^{t}}\left[\frac{D^{3} \tau}{D \mathbf{u}^{3}} \cdot \mathbf{u}_{(1)} \cdot \mathbf{u}_{(1)} \cdot \mathbf{w}+\frac{D^{2} \tau}{D \mathbf{u}^{2}} \cdot \mathbf{u}_{(2, a)} \cdot \mathbf{w}\right] d A=0
\end{aligned}
$$


and

$$
\begin{aligned}
\iiint_{\mathcal{B}}\left[\frac{\partial^{2} \psi}{\partial \mathbf{F}^{2}}: \nabla \mathbf{u}_{(2, p)}\right. & \left.: \nabla \mathbf{w}+\frac{\partial^{2} \psi}{\partial \mathbf{F} \partial \lambda}: \nabla \mathbf{w}\right] d V \\
& +\iint_{\partial \mathcal{B}^{t}}\left[\frac{D^{2} \tau}{D \mathbf{u}^{2}} \cdot \mathbf{u}_{(2, p)} \cdot \mathbf{w}+\frac{\partial}{\partial \lambda} \frac{D \tau}{D \mathbf{u}} \cdot \mathbf{w}\right] d A=0 .
\end{aligned}
$$

It may be observed that if, for an admissible $\mathbf{w},(37)$ and (38) are satisfied, then the first variation of the $\mathbf{u}_{(2)}$ terms in (36) in the direction $\mathbf{w}$ is zero. We obtain the Euler-Lagrange equations as follows. We further decompose $\mathbf{u}_{(2, a)}$ such that

$$
\mathbf{u}_{(2, a)}=\sum_{\alpha_{1}, \alpha_{2}, \alpha_{3}, \alpha_{4}} a_{\alpha_{1}, \alpha_{2}}^{\left(\gamma_{2}, \gamma_{3}\right)} a_{\alpha_{3}, \alpha_{4}}^{\left(\gamma_{2}, \gamma_{3}\right)} \mathbf{u}_{(2)}^{\left(\alpha_{1}, \alpha_{2}, \alpha_{3}, \alpha_{4}\right)}+\lambda_{(2)}^{\left(\gamma_{2}, \gamma_{3}\right)} \mathbf{u}_{(2, p)}
$$

where we sum over $\alpha_{1}, \alpha_{2}, \alpha_{3}, \alpha_{4} \in\{ \pm 1\}$. Here $\mathbf{u}_{(2)}^{\left(\alpha_{1}, \alpha_{2}, \alpha_{3}, \alpha_{4}\right)}$ is the solution of the linear system

$$
\begin{aligned}
-\nabla \cdot \mathbf{P}_{(2)}^{\left(\alpha_{1}, \alpha_{2}, \alpha_{3}, \alpha_{4}\right)} & =\mathbf{0}, \\
\mathbf{P}_{(2)}^{\left(\alpha_{1}, \alpha_{2}, \alpha_{3}, \alpha_{4}\right)} \cdot \mathbf{n} & =\frac{D \mathbf{t}}{D \mathbf{u}} \cdot \mathbf{u}_{(2)}^{\left(\alpha_{1}, \alpha_{2}, \alpha_{3}, \alpha_{4}\right)} \\
& +\frac{D^{2} \mathbf{t}}{D \mathbf{u}^{2}} \cdot \mathbf{u}_{(1)}^{\left(\alpha_{1} \gamma_{2}, \alpha_{2} \gamma_{3}\right)} \cdot \mathbf{u}_{(1)}^{\left(\alpha_{3} \gamma_{2}, \alpha_{4} \gamma_{3}\right)} \text { over } \partial \mathcal{B}^{t}, \\
\mathbf{u}_{(2)}^{\left(\alpha_{1}, \alpha_{2}, \alpha_{3}, \alpha_{4}\right)} & =\mathbf{0} \text { over } \partial \mathcal{B}^{u},
\end{aligned}
$$

where $\mathbf{n}$ is the unit normal and

$$
\begin{aligned}
& P_{(2)}^{\left(\alpha_{1}, \alpha_{2}, \alpha_{3}, \alpha_{4}\right) a b}=P_{(1) c^{d} u_{(2)}^{a b} u^{\left(\alpha_{1}, \alpha_{2}, \alpha_{3}, \alpha_{4}\right)_{c}}{ }_{\mid d d}} \\
& \quad+P_{(2) c}^{a b} e^{f} u_{(1)}^{\left(\alpha_{1} \gamma_{2}, \alpha_{2} \gamma_{3}\right)_{\mid d}} u_{(1)}^{\left(\alpha_{3} \gamma_{2}, \alpha_{4} \gamma_{3}\right)_{e}}{ }_{\mid f} .
\end{aligned}
$$

The existence and uniqueness of the solution $\mathbf{u}_{(2)}^{\left(\alpha_{1}, \alpha_{2}, \alpha_{3}, \alpha_{4}\right)}$ to the above equations follows from the fact that the homogeneous ordinary differential equation is elliptic and nondegenerate (because, as we noted in Section 3.1, we assume that the modes go unstable at different times). We find, by multiplying (40)-(42) by an arbitrary admissible displacement $\mathbf{w}$ and integrating, that

$$
\begin{gathered}
\iiint_{\mathcal{B}}\left[\frac{\partial^{2} \psi}{\partial \mathbf{F}^{2}}: \nabla \mathbf{u}_{(2)}^{\left(\alpha_{1}, \alpha_{2}, \alpha_{3}, \alpha_{4}\right)}: \nabla \mathbf{w}\right. \\
\left.\quad+\frac{\partial^{3} \psi}{\partial \mathbf{F}^{3}}: \nabla \mathbf{u}_{(1)}^{\left(\alpha_{1} \gamma_{2}, \alpha_{2} \gamma_{3}\right)}: \nabla \mathbf{u}_{(1)}^{\left(\alpha_{3} \gamma_{2}, \alpha_{4} \gamma_{3}\right)}: \nabla \mathbf{w}\right] d V \\
+\iint_{\partial \mathcal{B}^{t}}\left[\frac{D^{3} \tau}{D \mathbf{u}^{3}} \cdot \mathbf{u}_{(1)}^{\left(\alpha_{1} \gamma_{2}, \alpha_{2} \gamma_{3}\right)} \cdot \mathbf{u}_{(1)}^{\left(\alpha_{3} \gamma_{2}, \alpha_{4} \gamma_{3}\right)} \cdot \mathbf{w}+\frac{D^{2} \tau}{D \mathbf{u}^{2}} \cdot \mathbf{u}_{(2)}^{\left(\alpha_{1}, \alpha_{2}, \alpha_{3}, \alpha_{4}\right)} \cdot \mathbf{w}\right] d A \\
=0 .
\end{gathered}
$$

It may also be observed that $\mathbf{u}_{(2)}^{\left(\alpha_{1}, \alpha_{2}, \alpha_{3}, \alpha_{4}\right)} \in \mathcal{U}_{\left(\left(\alpha_{1}+\alpha_{3}\right) \gamma_{2},\left(\alpha_{2}+\alpha_{4}\right) \gamma_{3}\right)}$ and

$$
\mathbf{u}_{(2)}^{\left(\alpha_{1}, \alpha_{2}, \alpha_{3}, \alpha_{4}\right)}=\mathbf{u}_{(2)}^{\left(\alpha_{3}, \alpha_{4}, \alpha_{1}, \alpha_{2}\right)} .
$$


Furthermore $\mathbf{u}_{(2, p)}$ is a solution of the linear system

$$
\begin{aligned}
-\nabla \cdot \mathbf{P}_{(2, p)} & =\mathbf{0} \\
\mathbf{P}_{(2, p)} \cdot \mathbf{n} & =\frac{D \mathbf{t}}{D \mathbf{u}} \cdot \mathbf{u}_{(2, p)}+\frac{\Gamma_{0}}{\Gamma} \frac{\partial}{\partial \lambda} \mathbf{t} \text { over } \partial \mathcal{B}^{t}, \\
\mathbf{u}_{(2, p)} & =\frac{\partial}{\partial \lambda} \mathbf{v} \text { over } \partial \mathcal{B}^{u},
\end{aligned}
$$

where $\mathbf{n}$ is the unit normal and

$$
P_{(2, p)}^{a b}=P_{(1) c}^{a b}{ }^{d} u_{(2, p) \mid d}{ }^{c}+\frac{\partial}{\partial \lambda} P^{a b} .
$$

The existence and uniqueness of the solution $\mathbf{u}_{(2, p)}$ is a direct consequence of our assumption that the base state solution is differentiable with respect to $\lambda$ in Section 3. The solution $\mathbf{u}_{(2)}$ is a local minimum if $\left(\gamma_{2}, \gamma_{3}\right)$ are the first modes to go unstable. We find that the equations governing $\mathbf{u}_{(2)}^{\left(\alpha_{1}, \alpha_{2}, \alpha_{3}, \alpha_{4}\right)}$ reduce to ordinary differential equations in $x^{1}$ under the Fourier expansion. They may thus be solved used finite-difference methods. Often, particularly for high wavenumbers, these equations will be highly numerically unstable. Therefore, it might be necessary to employ special techniques. For example we employed the method of compound matrices in [19] (refer to Ng and Reid [25] for a thorough description).

\subsection{Determination of the Magnitude of the Buckle}

After substituting the solution for $\mathbf{u}_{(2)}$ back into (36), we obtain a polynomial in $\left\{a_{ \pm, \pm}^{\left(\gamma_{2}, \gamma_{3}\right)}\right\}$ and $\lambda_{(2)}$. We will determine $\left\{a_{ \pm, \pm}\right\}$by differentiating the polynomial with respect to these variables, so that (36) is stationary. However before we do this we must simplify (36), using the following two identities. We substitute $\mathbf{w}=\mathbf{u}_{(2, a)}$ into (37) to obtain

$$
\begin{gathered}
\iiint_{\mathcal{B}} \frac{\partial^{3} \psi}{\partial \mathbf{F}^{3}}: \nabla \mathbf{u}_{(1)}: \nabla \mathbf{u}_{(1)}: \nabla \mathbf{u}_{(2, a)} d V+\iint_{\partial \mathcal{B}^{t}} \frac{D^{3} \tau}{D \mathbf{u}^{3}} \cdot \mathbf{u}_{(1)} \cdot \mathbf{u}_{(1)} \cdot \mathbf{u}_{(2, a)} d A \\
=-\iiint_{\mathcal{B}} \frac{\partial^{2} \psi}{\partial \mathbf{F}^{2}}: \nabla \mathbf{u}_{(2, a)}: \nabla \mathbf{u}_{(2, a)} d V-\iint_{\mathcal{B}} \frac{D^{2} \tau}{D \mathbf{u}^{2}} \cdot \mathbf{u}_{(2, a)} \cdot \mathbf{u}_{(2, a)} d A
\end{gathered}
$$

We substitute $\mathbf{w}=\mathbf{u}_{(2)}$ into (38) to obtain

$$
\begin{aligned}
\iiint_{\mathcal{B}}\left[\frac{\partial^{2} \psi}{\partial \mathbf{F}^{2}}: \nabla \mathbf{u}_{(2, p)}\right. & \left.: \nabla \mathbf{u}_{(2)}+\frac{\partial^{2} \psi}{\partial \mathbf{F} \partial \lambda}: \nabla \mathbf{u}_{(2)}\right] d V \\
& +\iint_{\partial \mathcal{B}^{t}}\left[\frac{D^{2} \tau}{D \mathbf{u}^{2}} \cdot \mathbf{u}_{(2, p)} \cdot \mathbf{u}_{(2)}+\frac{d}{d \lambda} \frac{D \tau}{D \mathbf{u}} \cdot \mathbf{u}_{(2)}\right] d A=0 .
\end{aligned}
$$


Using (50) and (51), we find that (36) simplifies to

$$
\begin{aligned}
\frac{1}{24} W_{(4)}=\iiint_{\mathcal{B}} & {\left[-\frac{1}{8} \frac{\partial^{2} \psi}{\partial \mathbf{F}^{2}}: \nabla \mathbf{u}_{(2, a)}: \nabla \mathbf{u}_{(2, a)}\right.} \\
+ & \frac{1}{4} \lambda_{(2)}\left(\frac{\partial^{3} \psi}{\partial \mathbf{F}^{3}}: \nabla \mathbf{u}_{(2, p)}: \nabla \mathbf{u}_{(1)}: \nabla \mathbf{u}_{(1)}+\frac{\partial^{3} \psi}{\partial \mathbf{F}^{2} \partial \lambda}: \nabla \mathbf{u}_{(1)}: \nabla \mathbf{u}_{(1)}\right. \\
& \left.\left.+\frac{1}{24} \frac{\partial^{4} \psi}{\partial \mathbf{F}^{4}}: \nabla \mathbf{u}_{(1)}: \nabla \mathbf{u}_{(1)}: \nabla \mathbf{u}_{(1)}: \nabla \mathbf{u}_{(1)}\right)\right] d V \\
+\iint_{\partial \mathcal{B}^{t}} & {\left[-\frac{1}{8} \frac{D^{2} \tau}{D \mathbf{u}^{2}} \cdot \mathbf{u}_{(2, a)} \cdot \mathbf{u}_{(2, a)}+\frac{1}{24} \frac{D^{4} \tau}{D \mathbf{u}^{4}} \cdot \mathbf{u}_{(1)} \cdot \mathbf{u}_{(1)} \cdot \mathbf{u}_{(1)} \cdot \mathbf{u}_{(1)}\right.} \\
+ & \left.\frac{1}{4} \lambda_{(2)}\left(\frac{D^{3} \tau}{D \mathbf{u}^{3}} \cdot \mathbf{u}_{(2, p)} \cdot \mathbf{u}_{(1)} \cdot \mathbf{u}_{(1)}+\frac{\partial}{\partial \lambda} \frac{D^{2} \tau}{D \mathbf{u}^{2}} \cdot \mathbf{u}_{(1)} \cdot \mathbf{u}_{(1)}\right)\right] d A
\end{aligned}
$$

We note that, due to the assumptions in Section 3, each of these integrals $\iiint P d V$ may be written as

$$
\begin{aligned}
\iiint P d V=\int_{-x_{c}^{3}}^{x_{c}^{3}} \int_{-x_{c}^{2}}^{x_{c}^{2}} \int_{x_{c}^{1}}^{x_{+}^{1}} P \operatorname{det}\left(\mathbf{g}_{a b}\right) d x^{1} d x^{2} d x^{3} & \\
& =\left.4 x_{c}^{2} x_{c}^{3} \int_{x_{c}^{1}}^{x_{+}^{1}} P\right|_{(0,0)} \operatorname{det}\left(\mathbf{g}_{a b}\right) d x^{1},
\end{aligned}
$$

where $P$ is one of the terms in (52) and $\left.\right|_{(0,0)}$ denotes the projection of $P$ onto $\mathcal{U}_{(0,0)}$ (as defined in Section 3.1). This is because terms which are not in $\mathcal{U}_{(0,0)}$ integrate to zero. This means that the cubic terms in $\left\{a_{\alpha, \beta}^{\left(\gamma_{2}, \gamma_{3}\right)}\right\}$ are identically zero.

In order that (52) is stationary, we require that its derivative with respect to $a_{\alpha, \beta}^{\left(\gamma_{2}, \gamma_{3}\right)}$ (for $\alpha= \pm, \beta= \pm$ ) is zero. We obtain an equation of the form

$$
\frac{1}{24} \frac{\partial W_{(4)}}{\partial a_{\alpha, \beta}^{\left(\gamma_{2}, \gamma_{3}\right)}}:=d_{\alpha, \beta}^{\left(\gamma_{2}, \gamma_{3}\right)}:=g_{\alpha, \beta}^{\left(\gamma_{2}, \gamma_{3}\right)}+a_{-\alpha,-\beta}^{\left(\gamma_{2}, \gamma_{3}\right)} h_{\alpha, \beta}^{\left(\gamma_{2}, \gamma_{3}\right)} \lambda_{(2)}=0
$$

where $g_{\alpha, \beta}^{\left(\gamma_{2}, \gamma_{3}\right)}$ is cubic in $\left\{a_{ \pm \alpha, \pm \beta}^{\left(\gamma_{2}, \gamma_{3}\right)}\right\}$ and $h_{\alpha, \beta}^{\left(\gamma_{2}, \gamma_{3}\right)}$ is independent of $\left\{a_{ \pm \alpha, \pm \beta}^{\left(\gamma_{2}, \gamma_{3}\right)}\right\}$. The specific structure of $g_{\alpha, \beta}^{\left(\gamma_{2}, \gamma_{3}\right)}$ and $h_{\alpha, \beta}^{\left(\gamma_{2}, \gamma_{3}\right)}$ may be obtained as follows. Using (45), we find that

$$
\begin{aligned}
g_{\alpha, \beta}^{\left(\gamma_{2}, \gamma_{3}\right)}=\iiint_{\mathcal{B}} & {\left[\frac{1}{6} \frac{\partial^{4} \psi}{\partial \mathbf{F}^{4}}: \nabla \mathbf{u}_{(1)}: \nabla \mathbf{u}_{(1)}: \nabla \mathbf{u}_{(1)}: \nabla \mathbf{u}_{(1)}^{\left(\alpha \gamma_{2}, \beta \gamma_{3}\right)}\right.} \\
& \left.-\frac{1}{2} \sum_{\mu, \nu} a_{\mu, \nu}^{\left(\gamma_{2}, \gamma_{3}\right)} \frac{\partial^{2} \psi}{\partial \mathbf{F}^{2}}: \nabla \mathbf{u}_{(2, a)}: \nabla \mathbf{u}_{(2)}^{(\alpha, \beta, \mu, \nu)}\right] d V \\
+ & \iint_{\partial \mathcal{B}^{t}}\left[-\frac{1}{2} \sum_{\mu, \nu} a_{\mu, \nu}^{\left(\gamma_{2}, \gamma_{3}\right)} \frac{D^{2} \tau}{D \mathbf{u}^{2}} \cdot \mathbf{u}_{(2, a)} \cdot \mathbf{u}_{(2)}^{(\alpha, \beta, \mu, \nu)}\right. \\
+ & \left.\frac{1}{6} \frac{D^{4} \tau}{D \mathbf{u}^{4}} \cdot \mathbf{u}_{(1)} \cdot \mathbf{u}_{(1)} \cdot \mathbf{u}_{(1)} \cdot \mathbf{u}_{(1)}^{\left(\alpha \gamma_{2}, \beta \gamma_{3}\right)}\right] d A,
\end{aligned}
$$


and

$$
\begin{aligned}
h_{\alpha, \beta}^{\left(\gamma_{2}, \gamma_{3}\right)}=\frac{1}{2} \iiint_{\mathcal{B}}\left[\frac{\partial^{3} \psi}{\partial \mathbf{F}^{3}}: \nabla \mathbf{u}_{(2, p)}: \nabla \mathbf{u}_{(1)}^{\left(-\alpha \gamma_{2},-\beta \gamma_{3}\right)}: \nabla \mathbf{u}_{(1)}^{\left(\alpha \gamma_{2}, \beta \gamma_{3}\right)}\right. \\
\left.+\frac{\partial^{3} \psi}{\partial \mathbf{F}^{2} \partial \lambda}: \nabla \mathbf{u}_{(1)}^{\left(-\alpha \gamma_{2},-\beta \gamma_{3}\right)}: \nabla \mathbf{u}_{(1)}^{\left(\alpha \gamma_{2}, \beta \gamma_{3}\right)}\right] d V \\
+\iint_{\partial \mathcal{B}^{t}}\left[\frac{D^{3} \tau}{D \mathbf{u}^{3}} \cdot \mathbf{u}_{(2, p)} \cdot \mathbf{u}_{(1)}^{\left(\alpha \gamma_{2}, \beta \gamma_{3}\right)} \cdot \mathbf{u}_{(1)}^{\left(-\alpha \gamma_{2},-\beta \gamma_{3}\right)}\right. \\
\left.+\frac{\partial}{\partial \lambda} \frac{D^{2} \tau}{D \mathbf{u}^{2}} \cdot \mathbf{u}_{(1)}^{\left(\alpha \gamma_{2}, \beta \gamma_{3}\right)} \cdot \mathbf{u}_{(1)}^{\left(-\alpha \gamma_{2},-\beta \gamma_{3}\right)}\right] d A .
\end{aligned}
$$

It may be observed that $h_{+,+}^{\left(\gamma_{2}, \gamma_{3}\right)}=\bar{h}_{-,-}^{\left(\gamma_{2}, \gamma_{3}\right)}$. We now rearrange these expressions so that they are in a form that is more convenient for the boundary layer analysis.

We simplify (55) through (44) and the identity (23), finding that

$$
\begin{aligned}
g_{\alpha, \beta}^{\left(\gamma_{2}, \gamma_{3}\right)}=\iiint_{\mathcal{B}} & {\left[\frac{1}{6} \frac{\partial^{4} \psi}{\partial \mathbf{F}^{4}}: \nabla \mathbf{u}_{(1)}: \nabla \mathbf{u}_{(1)}: \nabla \mathbf{u}_{(1)}: \nabla \mathbf{u}_{(1)}^{\left(\alpha \gamma_{2}, \beta \gamma_{3}\right)}\right.} \\
& \left.+\frac{1}{2} \frac{\partial^{3} \psi}{\partial \mathbf{F}^{3}}: \nabla \mathbf{u}_{(1)}^{\left(\alpha \gamma_{2}, \beta \gamma_{3}\right)}: \nabla \mathbf{u}_{(1)}: \nabla \mathbf{u}_{(2)}\right] d V \\
& +\iint_{\partial \mathcal{B}^{t}} \frac{1}{2} \frac{D^{3} \tau}{D \mathbf{u}^{3}} \cdot \mathbf{u}_{(1)}^{\left(\alpha \gamma_{2}, \beta \gamma_{3}\right)} \cdot \mathbf{u}_{(1)} \cdot \mathbf{u}_{(2)} d A \\
& \quad \iiint_{\partial \mathcal{B}^{t}} \frac{1}{6} \frac{D^{4} \tau}{D \mathbf{u}^{4}} \cdot \mathbf{u}_{(1)} \cdot \mathbf{u}_{(1)} \cdot \mathbf{u}_{(1)} \cdot \mathbf{u}_{(1)}^{\left(\alpha \gamma_{2}, \beta \gamma_{3}\right)} d A
\end{aligned}
$$

This expression (57) is easier to use in the boundary layer analysis that will follow than (55). In fact ${ }^{4}(54)$ is equivalent to the Fredholm solvability condition for the $\mathrm{O}\left(\epsilon^{3}\right)$ component of the equation of equilibrium (4)-(6). Thus in fact (54) holds in more general circumstances when we cannot define an energy potential for the system. It may be observed that $g_{\alpha, \beta}^{\left(\gamma_{2}, \gamma_{3}\right)}$ is a sum of cubic terms in $\left\{a_{ \pm, \pm}^{\left(\gamma_{2}, \gamma_{3}\right)}\right\}$, but $h_{\alpha, \beta}^{\left(\gamma_{2}, \gamma_{3}\right)}$ is independent of $\left\{a_{ \pm, \pm}^{\left(\gamma_{2}, \gamma_{3}\right)}\right\}$. Both $g_{\alpha, \beta}^{\left(\gamma_{2}, \gamma_{3}\right)}$ and $h_{\alpha, \beta}^{\left(\gamma_{2}, \gamma_{3}\right)}$ are independent of $\lambda_{(2)}$. It can be observed that $d_{-\alpha,-\beta}^{\left(\gamma_{2}, \gamma_{3}\right)}$ is the complex conjugate of $d_{\alpha, \beta}^{\left(\gamma_{2}, \gamma_{3}\right)}$. Thus we only need to solve (54) in the case that $\{\alpha=+, \beta=+\}$ and $\{\alpha=+, \beta=-\}$.

The stability of the buckled state is governed by the eigenvalues of the Ja-

${ }^{4}$ See MacLaurin [18]. 
cobian $M_{\alpha \beta \zeta \sigma}=\frac{\partial^{2} W_{(4)}}{\partial a_{\alpha, \beta}^{\left(\gamma_{2}, \gamma_{3}\right)} \partial a_{\zeta, \sigma}^{\left(\gamma_{2}, \gamma_{3}\right)}}$. The components are given by

$$
\begin{aligned}
& M_{\alpha \beta \zeta \sigma}= \iiint_{\mathcal{B}}\left[\frac{1}{2} \frac{\partial^{4} \psi}{\partial \mathbf{F}^{4}}: \nabla \mathbf{u}_{(1)}: \nabla \mathbf{u}_{(1)}: \nabla \mathbf{u}_{(1)}^{\left(\zeta \gamma_{2}, \sigma \gamma_{3}\right)}: \nabla \mathbf{u}_{(1)}^{\left(\alpha \gamma_{2}, \beta \gamma_{3}\right)}\right. \\
&-\frac{1}{2} \frac{\partial^{2} \psi}{\partial \mathbf{F}^{2}}: \nabla \mathbf{u}_{(2, a)}: \nabla \mathbf{u}_{(2)}^{(\alpha, \beta, \zeta, \sigma)} \\
&\left.-\sum_{\mu_{1}, \ldots, \mu_{4}} a_{\mu_{1}, \mu_{2}}^{\left(\gamma_{2}, \gamma_{3}\right)} a_{\mu_{3}, \mu_{4}}^{\left(\gamma_{2}, \gamma_{3}\right)} \frac{\partial^{2} \psi}{\partial \mathbf{F}^{2}}: \nabla \mathbf{u}_{(2)}^{\left(\zeta, \sigma, \mu_{1}, \mu_{2}\right)}: \nabla \mathbf{u}_{(2)}^{\left(\alpha, \beta, \mu_{3}, \mu_{4}\right)}\right] d V \\
&+\iint_{\partial \mathcal{B}^{t}} {\left[-\frac{1}{2} \frac{D^{2} \tau}{D \mathbf{u}^{2}} \cdot \mathbf{u}_{(2, a)} \cdot \mathbf{u}_{(2)}^{(\alpha, \beta, \zeta, \sigma)}+\frac{1}{2} \frac{D^{4} \tau}{D \mathbf{u}^{4}} \cdot \mathbf{u}_{(1)} \cdot \mathbf{u}_{(1)} \cdot \mathbf{u}_{(1)}^{\left(\zeta \gamma_{2}, \sigma \gamma_{3}\right)} \cdot \mathbf{u}_{(1)}^{\left(\alpha \gamma_{2}, \beta \gamma_{3}\right)}\right.} \\
&\left.-\sum_{\mu_{1}, \ldots, \mu_{4}} a_{\mu_{1}, \mu_{2}}^{\left(\gamma_{2}, \gamma_{3}\right)} a_{\mu_{3}, \mu_{4}}^{\left(\gamma_{2}, \gamma_{3}\right)} \frac{D^{2} \tau}{D \mathbf{u}^{2}} \cdot \mathbf{u}_{(2)}^{\left(\zeta, \sigma, \mu_{1}, \mu_{2}\right)} \cdot \mathbf{u}_{(2)}^{\left(\alpha, \beta, \mu_{3}, \mu_{4}\right)}\right] d A \\
&+\lambda_{(2)} \delta(\alpha,-\zeta) \delta(\beta,-\sigma) h_{\alpha, \beta}, \quad(58)
\end{aligned}
$$

where $\delta\left(\epsilon_{1}, \epsilon_{2}\right)$ equals 1 if $\epsilon_{1}=\epsilon_{2}$ and zero otherwise and $\mu_{i} \in\{+,-\}$. If $\left(\gamma_{2}, \gamma_{3}\right)$ is the first mode to go unstable, and all of the eigenvalues of $\mathbf{M}$ are positive then the buckled state is stable. If at least one eigenvalue is negative, then the buckled state is unstable. If $\left(\gamma_{2}, \gamma_{3}\right)$ is not the first mode to go unstable, then, as we have already discussed above, the analysis is not as straightforward.

\subsection{Magnitude and Stability of the Buckle when $a_{+,-}$and $a_{-,+}$are Identically Zero}

In this section we assume $(28)$ holds, i.e. $\lambda^{\left(\gamma_{2}, \gamma_{3}\right)} \neq \lambda^{\left(\gamma_{2},-\gamma_{3}\right)}$. It follows as a consequence of this that $a_{+,-}$and $a_{-,+}$are identically zero. The above equations may be simplified though use of the fact that terms which are not in $\mathcal{U}_{0,0}$ (as defined in Section 3.1) integrate to zero as a consequence of the regularity of the geometry. We find that the strain energy may be written in the form

$$
W_{(4)}=\frac{1}{2} C^{\left(\gamma_{2}, \gamma_{3}\right)}\left(a_{+,+} a_{-,-}\right)^{2}+a_{+,+} a_{-,-} h_{+,+}^{\left(\gamma_{2}, \gamma_{3}\right)} \lambda_{(2)}
$$

for some real constant $C^{\left(\gamma_{2}, \gamma_{3}\right)}$ which satisfies $C^{\left(\gamma_{2}, \gamma_{3}\right)}=C^{\left(-\gamma_{2},-\gamma_{3}\right)}$ and may be determined from (52). Note that $h_{+,+}^{\left(\gamma_{2}, \gamma_{3}\right)}=h_{-,-}^{\left(\gamma_{2}, \gamma_{3}\right)}$. Since $a_{+,+}=\bar{a}_{-,-}$, we may consider $(59)$ to be a quadratic in $|a|:=\sqrt{a_{+,+} a_{-,-}}$. We set the derivative of (59) with respect to $|a|$ to zero and find

$$
\frac{d}{d|a|} W_{(4)}=2 C^{\left(\gamma_{2}, \gamma_{3}\right)}|a|^{3}+2|a| h_{+,+}^{\left(\gamma_{2}, \gamma_{3}\right)} \lambda_{(2)}=0
$$

which yields

$$
|a|^{2}=-\lambda_{(2)} \frac{h_{+,+}^{\left(\gamma_{2}, \gamma_{3}\right)}}{C^{\left(\gamma_{2}, \gamma_{3}\right)}}
$$

It may be observed that

$$
2 C^{\left(\gamma_{2}, \gamma_{3}\right)}|a|^{4}=a_{+,+}^{\left(\gamma_{2}, \gamma_{3}\right)} g_{+,+}^{\left(\gamma_{2}, \gamma_{3}\right)}=a_{-,-}^{\left(\gamma_{2}, \gamma_{3}\right)} g_{-,-}^{\left(\gamma_{2}, \gamma_{3}\right)}
$$


We find that the second derivative of the energy is

$$
\frac{d^{2}}{d|a|^{2}} W_{(4)}=6 C^{\left(\gamma_{2}, \gamma_{3}\right)}|a|^{2}+2 h_{+,+}^{\left(\gamma_{2}, \gamma_{3}\right)} \lambda_{(2)} .
$$

We substitute (61) and find

$$
\frac{d^{2}}{d|a|^{2}} W_{(4)}=-4 h_{+,+}^{\left(\gamma_{2}, \gamma_{3}\right)} \lambda_{(2)}
$$

This governs the stability of the buckled state: if it is positive, and if $\left(\gamma_{2}, \gamma_{3}\right)$ is the first mode to go unstable, the buckled state is stable. If it is negative, the buckled state is unstable. We require the right hand side of (61) to be positive for there to exist buckled states. We are thus presented with a pitchfork bifurcation.

We finish by proving that $h_{+,+}^{\left(\gamma_{2}, \gamma_{3}\right)} \leq 0$. The significance of this is that the nature of the buckle is determined by the sign of $C^{\left(\gamma_{2}, \gamma_{3}\right)}$. It is supercritical (and stable) if $C^{\left(\gamma_{2}, \gamma_{3}\right)}>0$, and it is subcritical (and unstable) if $C^{\left(\gamma_{2}, \gamma_{3}\right)}<0$. The reason that $h_{+,+}^{\left(\gamma_{2}, \gamma_{3}\right)} \leq 0$ is as follows.

$$
\begin{aligned}
& h_{+,+}^{\left(\gamma_{2}, \gamma_{3}\right)}=\frac{1}{2} \frac{\partial}{\partial \lambda} \iiint_{\mathcal{B}} \frac{\partial^{2} \psi}{\partial \mathbf{F}^{2}}: \nabla \mathbf{u}_{(1)}^{\left(-\gamma_{2},-\gamma_{3}\right)}: \nabla \mathbf{u}_{(1)}^{\left(\gamma_{2}, \gamma_{3}\right)} d V \\
&+\frac{1}{2} \frac{\partial}{\partial \lambda} \iint_{\partial \mathcal{B}^{t}} \frac{D^{2} \tau}{D \mathbf{u}^{2}} \cdot \mathbf{u}_{(1)}^{\left(\gamma_{2}, \gamma_{3}\right)} \cdot \mathbf{u}_{(1)}^{\left(-\gamma_{2},-\gamma_{3}\right)} d A .
\end{aligned}
$$

In fact $(65)$ is equal to $\frac{1}{4} \frac{d H}{d \lambda}$, where

$$
\begin{aligned}
& H(\lambda)= \\
& \quad \iiint_{\mathcal{B}} \frac{\partial^{2} \psi}{\partial \mathbf{F}^{2}}:\left(\nabla \mathbf{u}_{(1)}^{\left(\gamma_{2}, \gamma_{3}\right)}+\nabla \mathbf{u}_{(1)}^{\left(-\gamma_{2},-\gamma_{3}\right)}\right):\left(\nabla \mathbf{u}_{(1)}^{\left(\gamma_{2}, \gamma_{3}\right)}+\nabla \mathbf{u}_{(1)}^{\left(-\gamma_{2},-\gamma_{3}\right)}\right) d V \\
& \quad+\iint_{\partial \mathcal{B}^{t}} \frac{D^{2} \tau}{D \mathbf{u}^{2}} \cdot\left(\mathbf{u}_{(1)}^{\left(\gamma_{2}, \gamma_{3}\right)}+\mathbf{u}_{(1)}^{\left(-\gamma_{2},-\gamma_{3}\right)}\right) \cdot\left(\mathbf{u}_{(1)}^{\left(\gamma_{2}, \gamma_{3}\right)}+\mathbf{u}_{(1)}^{\left(-\gamma_{2},-\gamma_{3}\right)}\right) d A .
\end{aligned}
$$

This is because

$$
\begin{aligned}
\iiint_{\mathcal{B}} \frac{\partial^{2} \psi}{\partial \mathbf{F}^{2}}: \nabla \mathbf{u}_{(1)}^{\left(\gamma_{2}, \gamma_{3}\right)}: & \nabla \mathbf{u}_{(1)}^{\left(\gamma_{2}, \gamma_{3}\right)} d V= \\
& \iiint_{\mathcal{B}} \frac{\partial^{2} \psi}{\partial \mathbf{F}^{2}}: \nabla \mathbf{u}_{(1)}^{\left(-\gamma_{2},-\gamma_{3}\right)}: \nabla \mathbf{u}_{(1)}^{\left(-\gamma_{2},-\gamma_{3}\right)} d V=0
\end{aligned}
$$

as a consequence of the regularity of the geometry (and similarly for the other terms). However since the system is stable with respect to variations in $U_{\left(\gamma_{2}, \gamma_{3}\right)} \cup$ $U_{\left(-\gamma_{2},-\gamma_{3}\right)}$ for all $\lambda<\lambda^{\left(\gamma_{2}, \gamma_{3}\right)}$, it must be the case that for all $\lambda<\lambda^{\left(\gamma_{2}, \gamma_{3}\right)}, H>0$. Since $H=0$ when $\lambda=\lambda^{\left(\gamma_{2}, \gamma_{3}\right)}$, it must be the case that $h_{+,+}^{\left(\gamma_{2}, \gamma_{3}\right)} \leq 0$.

\section{Boundary Layer Analysis of The Eigenmode}

We assume that a boundary layer develops on the face $\partial \mathcal{B}^{*}$ of $\mathcal{B}$ given by $x^{1}=x_{c}^{1}$. What we mean by this is that, if $\left(\gamma_{2}, \gamma_{3}\right)=\varsigma\left(\gamma_{2}^{*}, \gamma_{3}^{*}\right)$ for some fixed 
$\left\{\gamma_{2}^{*}, \gamma_{3}^{*}\right\}$, then as $\varsigma \rightarrow \infty, \lambda^{\left(\gamma_{2}, \gamma_{3}\right)}$ converges to a finite constant. We denote this constant $\lambda_{(I)}^{\left(\gamma_{2}^{*}, \gamma_{3}^{*}\right)}$. In the sections to follow, we will perform a boundary layer analysis to determine $\lambda_{(I)}^{\left(\gamma_{2}^{*}, \gamma_{3}^{*}\right)}$, and also the leading order of $\mathbf{u}_{(1)}$ and $\mathbf{u}_{(2)}$.

\subsection{Assumptions Concerning the Incremental Elasticity Tensor}

We have already made assumptions about the nature of the elasticity tensor in Section 3. We now outline additional assumptions about the elasticity tensor which will make our boundary layer analysis easier. That is, we assume that $P^{a b}{ }_{c}^{d} \neq 0$ if and only if one of the following three conditions holds

$$
\begin{gathered}
a=b \text { and } c=d \\
a=c \text { and } b=d \\
a=d \text { and } b=c .
\end{gathered}
$$

These assumptions ensure that the eigenvalues of the matrix $\mathbf{D}$ to be defined in the next section come in \pm pairs.

We now give an example of a strain energy function and base state deformation which together ensure that (68)-(70), as well as the assumptions in Section 3 , are satisfied. We suppose that the base state deformation is coaxial with the basis vectors, i.e. $F^{a}{ }_{A} \neq 0$ only if $a=A$. Furthermore we suppose that the stress $\mathbf{P}$ can be written as a sum of terms of the form

$$
q_{1} \mathbf{p}_{1}+\ldots+q_{k} \mathbf{p}_{k},
$$

where $\left\{\mathbf{p}_{j}\right\}$ are polynomials in $\mathbf{F}$ and $\mathbf{F}^{T}$, and $\left\{q_{j}\right\}$ are scalar-valued functions of the symmetric invariants $\left\{I_{1}, I_{2}, I_{3}\right\}$ of $\mathbf{F}$. It can be demonstrated that the resulting incremental stress tensor satisfies (68)-(70).

One example where the stress tensor has the above form (71) is when the material is isotropic. This is because the first Piola-Kirchhoff stress tensor of an isotropic material may be written in the form

$$
\mathbf{P}=\alpha_{0} \mathbf{F}+\alpha_{1} \mathbf{F} \cdot \mathbf{C}+\alpha_{2} \mathbf{C}^{2},
$$

where $\mathbf{C}=\mathbf{F}^{T} \cdot \mathbf{F}$, and $\left\{\alpha_{0}, \alpha_{1}, \alpha_{2}\right\}$ are functions of the symmetric invariants of $\mathbf{F}$.

The stress tensor also has the above form (71) in the modelling of growing isotropic materials using a multiplicative decomposition of the deformation gradient (see Rodriguez et al. [27], Goriely et al. [11], MacLaurin et al. [19]). In brief, the deformation gradient is decomposed as $\mathbf{F}=\mathbf{F}_{e} \cdot \mathbf{F}_{g}$, where $\mathbf{F}_{g}$ is the growth tensor. We require the growth tensor $\mathbf{F}_{g}$ and deformation gradient $\mathbf{F}$ to be diagonal with respect to the basis vectors. If the material (which is isotropic) has strain energy function $\psi(\mathbf{F}, \mathbf{X}, \lambda)$, then the stress response is given by

$$
\mathbf{P}=\left.\operatorname{det}\left(\mathbf{F}_{g}\right) \frac{\partial \psi}{\partial \mathbf{F}}\right|_{\mathbf{F}_{e}} \mathbf{F}_{g}^{-T},
$$

which, through (72), may be seen to be of the form (71). 


\subsection{Determination of the Asymptotic Limit of $\lambda$}

Throughout this section, we perform a boundary layer analysis on the equations governing $\mathbf{u}_{(1)}^{\left(\gamma_{2}, \gamma_{3}\right)}$, i.e. (14), (18) and (19). We recall that $\mathbf{u}_{(1)}^{\left(\gamma_{2}, \gamma_{3}\right)}$ is of the form

$$
\mathbf{u}_{(1)}^{\left(\gamma_{2}, \gamma_{3}\right)}=\mathbf{Z}^{\left(\gamma_{2}, \gamma_{3}\right)} \exp \left(i\left(\gamma_{2} x^{2}+\gamma_{3} x^{3}\right)\right)
$$

We rescale $x^{1}$ as $x_{c}^{1}+\rho \varsigma^{-1}$ (recall that $x^{1}=x_{c}^{1}$ on $\partial \mathcal{B}^{*}$ ). This rescaling entails that, after a substitution of the above Fourier ansatz, and the substitutions $\gamma_{2}=\varsigma \gamma_{2}^{*}$ and $\gamma_{3}=\varsigma \gamma_{3}^{*}$, the field equation governing the onset of buckling (18) may be written as

$$
\left(\varsigma \frac{\partial}{\partial \rho} P_{(1)}^{a 1}+\varsigma i \gamma_{k}^{*} P_{(1)}^{a k}+P_{(1)}^{a l} \Gamma_{l b}^{b}+P_{(1)}^{l b} \Gamma_{l b}^{a}\right) e^{i \gamma_{2} x^{2}} e^{i \gamma_{3} x^{3}}=0 .
$$

We have, for convenience, dropped the $\left(\gamma_{2}, \gamma_{3}\right)$ superscripts from $\mathbf{Z}$, i.e. we write $\mathbf{Z}^{\left(\gamma_{2}, \gamma_{3}\right)}=\mathbf{Z}=\left(Z^{1}, Z^{2}, Z^{3}\right)^{T}$. We similarly drop the subscripts from $\mathbf{u}_{(1)}$. In the above summation (and for all following summations), we stipulate that $\gamma_{1}^{*}=0$, so that for example $\gamma_{k}^{*} P_{(1)}^{a k}=\gamma_{2}^{*} P_{(1)}^{a 2}+\gamma_{3}^{*} P_{(1)}^{a 3}$. We now outline the specific structure of (75), bearing in mind our assumptions (68)-(70). It has components of orders $\left\{\varsigma^{2}, \varsigma, 1\right\}$. We find the $a^{\prime}$ th component of the coefficient of $\varsigma^{2}$ is

$$
P^{a 1}{ }_{a}^{1} Z_{, \rho \rho}^{a}+i \gamma_{k}^{*} P^{a 1}{ }_{j}{ }^{k} Z_{, \rho}^{j}+i \gamma_{k}^{*} P^{a k}{ }_{j}{ }^{1} Z_{, \rho}^{j}-\gamma_{k}^{*} \gamma_{m}^{*} P^{a k}{ }_{j}{ }^{m} Z^{j} .
$$

The $a$ 'th component of the coefficient of $\varsigma Z_{, \rho}^{j}$ is

$$
\frac{d}{d x^{1}}\left(P_{a}^{a 1}{ }^{1}\right) \delta_{j}^{a}+P_{m}^{a 1}{ }^{n} \Gamma_{n j}^{m}+P_{j}^{a l}{ }_{j}^{1} \Gamma_{l b}^{b}+P_{j}^{l b}{ }_{j}^{1} \Gamma_{l b}^{a} \text {. }
$$

The $a^{\prime}$ th component of the coefficient of $\varsigma Z^{j}$ is

$$
i \gamma_{k}^{*} \frac{d}{d x^{1}} P_{j}^{a 1{ }_{j}}+i \gamma_{k}^{*} \Gamma_{m j}^{n} P_{n}^{a k}{ }^{m}+i \gamma_{k}^{*} \Gamma_{l b}^{b} P_{j}^{a l}{ }^{k}+i \gamma_{k}^{*} P_{j}^{l b}{ }_{j}^{k} \Gamma_{l b}^{a} .
$$

The $a^{\prime}$ th component of the coefficient of $Z^{j}$ is

$$
\frac{\partial}{\partial x^{1}}\left(P_{m}^{a 1}{ }^{n}\right) \Gamma_{n j}^{m}+P_{m}^{a l}{ }^{n} \Gamma_{l b}^{b} \Gamma_{n j}^{m}+P_{m}^{l b}{ }^{n} \Gamma_{l b}^{a} \Gamma_{n j}^{m}
$$

Let $\mathbf{V}=\left(Z^{1}, Z^{2}, Z^{3}, Z_{, \rho}^{1}, Z_{, \rho}^{2}, Z_{, \rho}^{3}\right)^{T}$. We may then write $(75)$ in the form

$$
\varsigma^{2}\left(\mathbf{A}^{(0)} \cdot \frac{\partial \mathbf{V}}{\partial \rho}+\mathbf{A}^{(1)} \cdot \mathbf{V}\right)+\varsigma \mathbf{A}^{(2)} \cdot \mathbf{V}+\mathbf{A}^{(3)} \cdot \mathbf{V}=0 .
$$

Here $\left\{\mathbf{A}^{(j)}\right\}$ are $6 \times 6$ matrices. We stipulate the $(j+3)$ 'th row of $(80)$ (where $j \in\{1,2,3\})$ to derive from the identity $\frac{\partial V_{j}}{\partial \rho}=V_{j+3}$. The structure of the first three rows of these matrices can be inferred from (76)-(79). Indeed, it may be inferred from (76)-(79) and the coaxiality assumptions (68)-(70) that the structures of $\mathbf{A}^{(0)}$ and $\mathbf{A}^{(1)}$ are given by (where an ' $x^{\prime}$ denotes a potentially 
nonzero number)

$$
\mathbf{A}^{(0)}=\left(\begin{array}{llllll}
0 & 0 & 0 & x & 0 & 0 \\
0 & 0 & 0 & 0 & x & 0 \\
0 & 0 & 0 & 0 & 0 & x \\
x & 0 & 0 & 0 & 0 & 0 \\
0 & x & 0 & 0 & 0 & 0 \\
0 & 0 & x & 0 & 0 & 0
\end{array}\right), \mathbf{A}^{(1)}=\left(\begin{array}{cccccc}
x & 0 & 0 & 0 & x & x \\
0 & x & x & x & 0 & 0 \\
0 & x & x & x & 0 & 0 \\
0 & 0 & 0 & x & 0 & 0 \\
0 & 0 & 0 & 0 & x & 0 \\
0 & 0 & 0 & 0 & 0 & x
\end{array}\right) .
$$

We perform the following asymptotic expansion of $\mathbf{V}$ and $\lambda$ over the boundary layer,

$$
\begin{aligned}
\lambda & =\lambda_{(I)}^{\left(\gamma_{2}^{*}, \gamma_{3}^{*}\right)}+\varsigma^{-1} \lambda_{(I I)}^{\left(\gamma_{2}^{*}, \gamma_{3}^{*}\right)}+\ldots \\
\mathbf{V} & =\mathbf{V}_{(I)}+\varsigma^{-1} \mathbf{V}_{(I I)}+\ldots
\end{aligned}
$$

Our aim in this section is to determine the leading order terms of $\lambda$ and $\mathbf{V}$.

It is a consequence of our ellipticity assumption (25) that each of the elements denoted by an ' $\mathrm{x}$ ' in the expression for $\mathbf{A}^{(0)}$ in $(81)$ are nonzero, which means that $\mathbf{A}^{(0)}$ is invertible. It may be readily inferred that the eigenvalues of $\left(\mathbf{A}^{(0)}\right)^{-1} \cdot \mathbf{A}^{(1)}$ occur in \pm pairs. We assume that they all have nonzero real part, and that we have the following diagonalisation ${ }^{5}$

$$
\left(\mathbf{A}^{(0)}\right)^{-1} \cdot \mathbf{A}^{(1)}=-\mathbf{E} \cdot \mathbf{D} \cdot \mathbf{E}^{-1}
$$

We assume the first three eigenvalues of $-\mathbf{D}$ have negative real part, and the last three eigenvalues have positive real part. We find

$\varsigma^{2} \mathbf{E}^{-1} \cdot \frac{\partial \mathbf{V}}{\partial \rho}=\varsigma^{2} \mathbf{D} \cdot \mathbf{E}^{-1} \cdot \mathbf{V}-\varsigma \mathbf{E}^{-1} \cdot\left(\mathbf{A}^{(0)}\right)^{-1} \cdot \mathbf{A}^{(2)} \cdot \mathbf{V}-\mathbf{E}^{-1} \cdot\left(\mathbf{A}^{(0)}\right)^{-1} \cdot \mathbf{A}^{(3)} \cdot \mathbf{V}$.

We are careful to consistently order the eigenvalues such that $\mathbf{D}$ and $\mathbf{E}$ are continuous and differentiable functions of $x^{1}$ and $\lambda$. We stipulate that the norm of each column ${ }^{6}$ of $\mathbf{E}$ is 1 . We let $\mathbf{Y}=\mathbf{E}^{-1} \cdot \mathbf{V}$, and we perform the following asymptotic expansion

$$
\begin{aligned}
& \mathbf{Y}=\mathbf{Y}_{(I)}+\varsigma^{-1} \mathbf{Y}_{(I I)}+\ldots \\
& \mathbf{E}=\mathbf{E}_{(I)}+\varsigma^{-1} \mathbf{E}_{(I I)}+\ldots \\
& \mathbf{D}=\mathbf{D}_{(I)}+\varsigma^{-1} \mathbf{D}_{(I I)}+\ldots
\end{aligned}
$$

We outline further below our method for determining $\mathbf{Y}_{(I)}, \mathbf{E}_{(I)}$ and $\mathbf{D}_{(I)}$. We find that (85) may be simplified to

$$
\varsigma^{2} \frac{\partial \mathbf{Y}}{\partial \rho}=\varsigma^{2} \mathbf{D} \cdot \mathbf{Y}+\varsigma \mathbf{B}^{(1)} \cdot \mathbf{Y}+\mathbf{B}^{(2)} \cdot \mathbf{Y}
$$

\footnotetext{
${ }^{5} \mathrm{~A}$ sufficient condition for the existence of the diagonalisation is that all the eigenvalues are distinct.

${ }^{6}$ Note that rescaling the columns of $\mathbf{E}$ does not affect our results.
} 
where

$$
\begin{aligned}
& \mathbf{B}^{(1)}=-\mathbf{E}^{-1} \cdot\left(\mathbf{A}^{(0)}\right)^{-1} \cdot \mathbf{A}^{(2)} \cdot \mathbf{E}+\frac{d}{d R}\left(\mathbf{E}^{-1}\right) \cdot \mathbf{E}, \\
& \mathbf{B}^{(2)}=-\mathbf{E}^{-1} \cdot\left(\mathbf{A}^{(0)}\right)^{-1} \cdot \mathbf{A}^{(3)} \cdot \mathbf{E} .
\end{aligned}
$$

The leading order of the solution of (86) is

$$
Y_{(I)}^{j}=C^{j} \exp \left(d_{j j} \rho\right)
$$

for some constants $\left\{C^{j}\right\}$. Since the real components of $\left\{d_{44}, d_{55}, d_{66}\right\}$ are greater than zero, we stipulate $\left\{C^{4}, C^{5}, C^{6}\right\}$ to be equal to zero to ensure that the solution is asymptotically decaying. We thus find that

$$
V_{(I)}^{k}=E_{k j} C^{j} \exp \left(d_{j j} \rho\right) .
$$

The leading order of the displacement gradient is given by (for $b \in\{1,2,3\}$ )

$$
\begin{aligned}
u_{(1)}{ }^{b}{ }_{m} & =\varsigma \sum_{j=1}^{3} u_{(1, j)}^{*}{ }_{m}{ }_{m} \exp \left(\rho d_{j j}\right) \exp \left(i \varsigma\left(\gamma_{2}^{*} x^{2}+\gamma_{3}^{*} x^{3}\right)\right) \text { where, } \\
u_{(1, j)}^{*}{ }^{b}{ }_{1} & =d_{j j} E_{b j} C^{j}, \\
u_{(1, j)}^{*}{ }^{b}{ }_{m} & =i \gamma_{m}^{*} E_{b j} C^{j} \text { where } m=2 \text { or } m=3 .
\end{aligned}
$$

The boundary condition at $\partial \mathcal{B}^{*}$ could be either that of pressure or null-displacement. The incremental stress boundary condition (19) at $\partial \mathcal{B}^{*}$ is, to leading order,

$$
\varsigma P^{a 1}{ }_{b}^{c} u_{(1)}{ }^{b}{ }_{c}=0, \text { for all } a \in\{1,2,3\}
$$

where the above expression is evaluated at $\rho=0$. We note that $\frac{D \tau}{D \mathbf{u}}$ is not in this expression because, being independent of $\nabla \mathbf{u}_{(1)}$, it has lower asymptotic order. Alternatively, the incremental displacement boundary condition (14) at $\partial \mathcal{B}^{*}$ is

$$
V_{(I)}^{a}=0 \text { for all } a \in\{1,2,3\} .
$$

Whichever boundary condition applies, we obtain the following condition

$$
\mathbf{Q} \cdot \mathbf{C}=\mathbf{0},
$$

where $\mathbf{C}=\left(C^{1}, C^{2}, C^{3}\right)^{T}$ and $\mathbf{Q}$ is a $3 \times 3$ matrix which can be inferred from (90)-(95). For there to exist a boundary layer, we thus require

$$
\operatorname{det}(\mathbf{Q})=0 .
$$

This is the condition we use to solve for the leading order of the critical parameter, i.e. $\lambda_{(I)}^{\left(\gamma_{2}^{*}, \gamma_{3}^{*}\right)}$. We assume that when (97) is satisfied, the kernel of $\mathbf{Q}$ is of one dimension only. The condition (97) is analogous to the "complementing condition' of Negron-Marrero and Montes-Pizarro [23, 24]. It is interesting to observe that (97) is independent of the Christoffel symbols, which means that $\lambda_{(I)}^{\left(\gamma_{2}^{*}, \gamma_{3}^{*}\right)}$ is independent of the local geometry (as has been pointed out by Hohlfeld and 
Mahadevan [14] and Negron-Marrero and Montes-Pizarro [23]).

\subsection{Solution of $\lambda_{(I I)}^{\left(\gamma_{2}^{*}, \gamma_{3}^{*}\right)}$}

We determine the next order of $\lambda$, i.e. $\lambda_{(I I)}^{\left(\gamma_{2}^{*}, \gamma_{3}^{*}\right)}$, by solving the $\mathrm{O}(1)$ component of (86), i.e.

$$
\begin{aligned}
\frac{\partial}{\partial \rho} Y_{(I I)}^{j}=d_{j j} Y_{(I I)}^{j}+B_{j k}^{(1)} C^{k} \exp ( & \left.\rho d_{k k}\right)+ \\
& \left(\lambda_{(I I)}^{\left(\gamma_{2}^{*}, \gamma_{3}^{*}\right)} \frac{\partial d_{j j}}{\partial \lambda}+\rho \frac{\partial d_{j j}}{\partial R}\right) C^{j} \exp \left(\rho d_{j j}\right) .
\end{aligned}
$$

We make use of the following asymptotic expansions for $\mathbf{E}_{(I I)}$ and $\mathbf{D}_{(I I)}$,

$$
\begin{aligned}
& \mathbf{D}_{(I I)}=\rho \frac{d}{d R} \mathbf{D}+\lambda_{(I I)}^{\left(\gamma_{2}^{*}, \gamma_{3}^{*}\right)} \frac{d}{d \lambda} \mathbf{D} \\
& \mathbf{E}_{(I I)}=\rho \frac{d}{d R} \mathbf{E}+\lambda_{(I I)}^{\left(\gamma_{2}^{*}, \gamma_{3}^{*}\right)} \frac{d}{d \lambda} \mathbf{E} .
\end{aligned}
$$

The derivatives are always evaluated at $\lambda=\lambda_{(I)}^{\left(\gamma_{2}^{*}, \gamma_{3}^{*}\right)}$. The general solution of (98) is

$$
\begin{aligned}
Y_{(I I)}^{j} & =C^{k} B_{j k}^{(1)}\left(d_{k k}-d_{j j}\right)^{-1} \exp \left(\rho d_{k k}\right)+ \\
& C^{j}\left(B_{j j}^{(1)}+\lambda_{(I I)}^{\left(\gamma_{2}^{*}, \gamma_{3}^{*}\right)} \frac{\partial d_{j j}}{\partial \lambda}+\frac{\rho}{2} \frac{\partial d_{j j}}{\partial \rho}\right) \rho \exp \left(\rho d_{j j}\right)+C_{(I I)}^{j} \exp \left(\rho d_{j j}\right),
\end{aligned}
$$

where $j \in\{1, \ldots, 6\}, \mathbf{C}_{(I I)}$ is a vector of constants and we sum over all $k \neq j$. Note that $C_{(I I)}^{j}$ must be identically zero if $\operatorname{Re}\left(d_{j j}\right)>0$ to ensure that the solution decays. At $\rho=0$,

$$
Y_{(I I)}^{j}=C^{k} B_{j k}^{(1)}\left(d_{k k}-d_{j j}\right)^{-1}+C_{(I I)}^{j},
$$

where we sum over all $k \neq j$.

We determine $\lambda_{(I I)}^{\left(\gamma_{2}^{*}, \gamma_{3}^{*}\right)}$ by matching the next order of the boundary condition, which could be either null-stress (19) or null-displacement (14). We begin by considering the null-displacement boundary condition $Z^{a}=0$ (for all $a \in\{1,2,3\})$ at $\rho=0$. At $O(1)$, this may be written as

$$
\mathbf{V}_{(I I)}=\mathbf{E} \cdot \mathbf{Y}_{(I I)}+\frac{\partial}{\partial \lambda} \mathbf{E} \cdot \mathbf{Y}_{(I)}=\mathbf{0} .
$$

We find, for all $a \in\{1,2,3\}$,

$$
q^{a}+E_{a b} C^{c} B_{b c}^{(1)}\left(d_{c c}-d_{b b}\right)^{-1}+\left(\frac{\partial}{\partial \lambda} E_{a b}\right) C^{b} \lambda_{(I I)}^{\left(\gamma_{2}^{*}, \gamma_{3}^{*}\right)}=0,
$$

where $\mathbf{q}=\mathbf{Q} \cdot \mathbf{C}_{(I I)}$ and we sum over all $b \neq c$. We assumed earlier that the kernel of $\mathbf{Q}$ is of one dimension, which means that the range of $\mathbf{Q}$ must be of dimension 2. We dot multiply (104) with a vector perpendicular to the range of $\mathbf{Q}$ to eliminate $\mathbf{q}$ and determine $\lambda_{(I I)}^{\left(\gamma_{2}^{*}, \gamma_{3}^{*}\right)}$. 
We now consider the stress boundary condition (19). We firstly write $\nabla \mathbf{u}$ to its leading two orders, i.e.

$$
u_{\mid a}^{b}=\left(\varsigma \sum_{j=1}^{3} u_{(1, j)}^{*}{ }_{a}^{b}+\lambda_{(I I)}^{\left(\gamma_{2}^{*}, \gamma_{3}^{*}\right)} m_{a}^{b}{ }_{a}+n^{b}{ }_{a}\right) e^{i \gamma_{2} x^{2}} e^{i \gamma_{3} x^{3}}
$$

where, for $a \in\{2,3\}$ and $b \in\{1,2,3\}$,

$$
\begin{aligned}
m^{b}{ }_{1} & =C^{j} \frac{\partial}{\partial \lambda} E_{(b+3) j} \\
m^{b}{ }_{a} & =i \gamma_{a}^{*} C^{j} \frac{\partial}{\partial \lambda} E_{b j} \\
n^{b}{ }_{1} & =B_{j k}^{(1)} C^{k}\left(d_{k k}-d_{j j}\right)^{-1} E_{(b+3) j}+\Gamma_{c 1}^{b} C^{k} E_{c k}+C_{(I I)}^{k} E_{(b+3) k} \\
n^{b}{ }_{a} & =i \gamma_{a}^{*} B_{j k}^{(1)}\left(d_{k k}-d_{j j}\right)^{-1} C^{k} E_{b j}+\Gamma_{c a}^{b} C^{k} E_{c k}+i \gamma_{a}^{*} C_{(I I)}^{k} E_{b k},
\end{aligned}
$$

where we sum over all $k \neq j$. We recall that $\mathbf{u}_{(1, j)}^{*}$ is defined in (92)-(93). We thus find the $O(1)$ component of the boundary condition to be

$$
\begin{aligned}
& q^{a}+P_{c}^{a 1}{ }_{c}^{d} n_{d}^{c}-\frac{\Gamma_{0}}{\Gamma} \frac{d t^{a}}{d u^{b}} E_{b k} C^{k}+ \\
& \lambda_{(I I)}^{\left(\gamma_{2}^{*}, \gamma_{3}^{*}\right)}\left(\frac{\partial}{\partial \lambda} P^{a 1}{ }_{c}{ }^{d} \sum_{j=1}^{3} u_{(1, j)}^{*}{ }^{c}{ }_{d}+P^{a 1}{ }_{c}{ }^{d} m_{d}^{c}{ }_{d}\right)=0,
\end{aligned}
$$

where $\mathbf{q}=\mathbf{Q} \cdot \mathbf{C}_{(I I)}$. We eliminate $\mathbf{q}$ by dot multiplying (110) with a vector perpendicular to the range of $\mathbf{Q}$ to solve for $\lambda_{(I I)}^{\left(\gamma_{2}^{*}, \gamma_{3}^{*}\right)}$.

\subsection{Structure of $\mathbf{u}_{(1)}$}

The above boundary layer analysis was performed on $\mathbf{u}_{(1)}^{\left(\gamma_{2}, \gamma_{3}\right)}$. We now investigate how altering the signs of $\gamma_{2}$ and $\gamma_{3}$ affects the analysis. We may directly infer from $(27)$ that $\lambda^{\left(-\gamma_{2}^{*},-\gamma_{3}^{*}\right)}=\lambda^{\left(\gamma_{2}^{*}, \gamma_{3}^{*}\right)}$, and $\mathbf{u}_{(1)}^{\left(-\gamma_{2},-\gamma_{3}\right)}=\overline{\mathbf{u}}_{(1)}^{\left(\gamma_{2}, \gamma_{3}\right)}$.

We now demonstrate that

$$
\lambda_{(I)}^{\left(\gamma_{2}^{*}, \gamma_{3}^{*}\right)}=\lambda_{(I)}^{\left(-\gamma_{2}^{*}, \gamma_{3}^{*}\right)}
$$

This is for the following reasons. Firstly, $\mathbf{D}$ is unchanged when $\gamma_{2}^{*} \rightarrow-\gamma_{2}^{*}$, and the second and fifth rows of $\mathbf{E}$ switch signs. In the case of the null-stress boundary condition, it can be seen that $\mathbf{Q}$ is unchanged, except $\left\{Q_{12}, Q_{21}, Q_{23}, Q_{32}\right\}$ switch signs. In the case of the null-displacement boundary condition, $Q_{2 a} \rightarrow$ $-Q_{2 a}$. Thus in both cases, the condition (97) governing $\lambda_{(I)}^{\left(\gamma_{2}^{*}, \gamma_{3}^{*}\right)}$ also governs $\lambda_{(I)}^{\left(-\gamma_{2}^{*}, \gamma_{3}^{*}\right)}$. In turn, it follows from (27) and (111) that

$$
\lambda_{(I)}^{\left(\gamma_{2}^{*}, \gamma_{3}^{*}\right)}=\lambda_{(I)}^{\left(-\gamma_{2}^{*}, \gamma_{3}^{*}\right)}=\lambda_{(I)}^{\left(\gamma_{2}^{*},-\gamma_{3}^{*}\right)}=\lambda_{(I)}^{\left(-\gamma_{2}^{*},-\gamma_{3}^{*}\right)}
$$

However it is not necessarily the case that

$$
\lambda_{(I I)}^{\left(\gamma_{2}^{*}, \gamma_{3}^{*}\right)}=\lambda_{(I I)}^{\left(-\gamma_{2}^{*}, \gamma_{3}^{*}\right)}
$$


It can be shown that a sufficient condition for (113) is

$$
\tilde{\mathbf{I}} \cdot \mathbf{A}^{(2)} \cdot \tilde{\mathbf{I}}=\mathbf{A}^{(2)},
$$

where $\tilde{\mathbf{I}}$ is equal to the identity, except $\tilde{I}_{2,2}=\tilde{I}_{5,5}=-1$. Note that, due to (27), $\lambda_{(I I)}^{\left(\gamma_{2}^{*}, \gamma_{3}^{*}\right)}=\lambda_{(I I)}^{\left(\gamma_{2}^{*},-\gamma_{3}^{*}\right)}$ if and only if (113) is satisfied. Furthermore if (114) is satisfied, a sufficient condition that $\lambda_{(j)}^{\left(\gamma_{2}^{*}, \gamma_{3}^{*}\right)}=\lambda_{(j)}^{\left(-\gamma_{2}^{*}, \gamma_{3}^{*}\right)}($ for $j \geq I I I)$, is

$$
\tilde{\mathbf{I}} \cdot \mathbf{A}^{(3)} \cdot \tilde{\mathbf{I}}=\mathbf{A}^{(3)} .
$$

\section{Boundary Layer Analysis of Postbuckled Solution}

We determine the leading asymptotic behaviour of $\left\{\mathbf{u}_{(2)}^{\left(\alpha_{1}, \alpha_{2}, \alpha_{3}, \alpha_{4}\right)}\right\}$ as $\varsigma \rightarrow$ $\infty$. These variables are defined in Section 4.1. Once we have determined the asymptotic behaviour of these variables, we determine the asymptotic behaviour of the integrals governing the magnitude and stability of the buckle.

\subsection{Solution of $\mathbf{u}_{(2, a)}$}

It is convenient to define a new boundary layer variable $\nu$ so that the boundary layer equations have a similar form to the previous section. We let $x^{1}=x_{c}^{1}+\frac{1}{2} \varsigma^{-1} \nu$, so that $\nu=2 \rho$. We consider that $\alpha_{j}=+$ for all $j$ throughout this section (we give reasons for this assumption further below). We define $\mathbf{Z}$ and $\mathbf{V}$ analogously to Section 5, i.e.

$$
\begin{aligned}
u_{(2)}^{(+,+,+,+) a} & =Z^{a}\left(x^{1}\right) \exp \left(i \varsigma\left(2 \gamma_{2}^{*} x^{2}+2 \gamma_{3}^{*} x^{3}\right)\right) \\
\mathbf{V} & =\left(Z^{1}, Z^{2}, Z^{3}, Z_{, \nu}^{1}, Z_{, \nu}^{2}, Z_{, \nu}^{3}\right)^{T} .
\end{aligned}
$$

The $\nu$ subscript denotes differentiation with respect to $\nu$. We must be careful not to confuse $\mathbf{Z}, \mathbf{V}$ and $\mathbf{Y}$ with their definitions in the previous section. However it will be seen that $\mathbf{D}, \mathbf{E}$ and $\left\{C^{k}\right\}$ remain the same.

We find the leading order of the field equation (40) which governs $\mathbf{u}_{(2)}^{\left(\alpha_{1}, \ldots, \alpha_{4}\right)}$ over the boundary layer to be

$$
\begin{aligned}
(2 \varsigma)^{2}\left(\mathbf{A}^{(0)} \cdot \frac{\partial \mathbf{V}}{\partial \nu}+\mathbf{A}^{(1)} \cdot \mathbf{V}\right) & +2 \varsigma \mathbf{A}^{(2)} \cdot \mathbf{V}+\mathbf{A}^{(3)} \cdot \mathbf{V} \\
& +(2 \varsigma)^{3} \sum_{1 \leq j, k \leq 3} \mathbf{A}_{(j, k)}^{(4)} \exp \left(\frac{\nu}{2}\left(d_{j j}+d_{k k}\right)\right)=\mathbf{0} .
\end{aligned}
$$

Here $\mathbf{A}^{(4)}$ is essentially obtained by substituting the asymptotic expression for $\mathbf{u}_{(1)}$ we obtained in Section 5 into $\nabla \cdot\left(\frac{\partial^{2} \mathbf{P}}{\partial \mathbf{F}^{2}}: \nabla \mathbf{u}_{(1)}: \nabla \mathbf{u}_{(1)}\right)$. We only need to keep the leading order terms of order $O\left((2 \varsigma)^{3}\right)$. More precisely, $A_{j k}^{(4) a}=0$ if $a \in\{4,5,6\}$, and if $a \in\{1,2,3\}$,

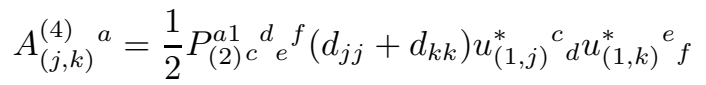

$$
\begin{aligned}
& +i \gamma_{m}^{*} P_{(2) c e^{d}{ }^{f} u_{(1, j)}^{*}{ }^{c} u_{(1, k)}^{*}{ }^{e} f}
\end{aligned}
$$


where $\mathbf{u}_{(1, j)}^{*}$ is defined in (92)-(93).

In the case of a null-stress boundary condition (19), we may write the leading order of the boundary condition at $\nu=0$ as

$$
\begin{aligned}
& P_{{ }^{a}{ }^{d} u_{(2)}{ }^{c} d}+\exp \left(i \nu\left(\gamma_{2}^{*} x^{2}+\gamma_{3}^{*} x^{3}\right)\right) \times \\
& \qquad P_{(2){ }^{c} e^{d}{ }^{f} \sum_{j, k=1}^{3} u_{(1, j)}^{*}{ }^{c}{ }^{d} u_{(1, k)}^{*}{ }^{e} f}=0,
\end{aligned}
$$

In the case of a null-displacement boundary condition (14), we may write the leading order of the boundary condition at $\nu=0$ as

$$
\mathbf{u}_{(2)}=\mathbf{0} .
$$

In the discussion to follow below, the 'homogeneous solution' of the above equations governing $\mathbf{u}_{(2)}$ is that obtained by setting $\mathbf{u}_{(1)}=0$. We have seen in Section 5 that the leading order of the homogeneous part of the field equation (118), i.e.

$$
\left(\mathbf{A}^{(0)} \cdot \frac{\partial \mathbf{V}}{\partial \nu}+\mathbf{A}^{(1)} \cdot \mathbf{V}\right)=\mathbf{0},
$$

together with the leading order of the homogeneous part of the boundary condition $((120)$ or $(121))$, has a nontrivial solution over the boundary layer. This means that, if $\mathbf{V}$ has leading order $(2 \varsigma)$, then in general we will not be able to find a solution to the leading order of (118) and ((120) or (121)). We therefore require that $\mathbf{V}$ has leading order $(2 \varsigma)^{2}$. Thus

$$
\mathbf{V}=(2 \varsigma)^{2} \mathbf{V}_{(I)}+(2 \varsigma) \mathbf{V}_{(I I)}+\ldots
$$

It can similarly be demonstrated that $\mathbf{u}_{(2)}^{(-,-,-,-)}$has leading order $(2 \varsigma)^{2}$. Indeed, since $\lambda_{(I)}^{\left(\gamma_{2},{ }^{*},{ }_{3}^{*}\right)}=\lambda_{(I)}^{\left(-\gamma_{2},{ }^{*}, \gamma_{3}^{*}\right)}$, we may also determine $\mathbf{u}_{(2)}^{(+,-,+,-)}$and $\mathbf{u}_{(2)}^{(-,+,-,+)}$, and these have leading order $(2 \varsigma)^{2}$. However in general circumstances we expect the other variables to have leading order $(2 \varsigma)$. To see this, consider for example $\mathbf{u}_{(2)}^{(+,+,-,-)} \in \mathcal{U}_{0,0}$. The homogeneous equation governing $\mathbf{u}_{(2)}^{(+,+,-,-)}$may be obtained by taking the homogeneous equation governing $\mathbf{u}_{(1)}$ (i.e. 80 ) and substituting $\gamma_{2}^{*}=\gamma_{3}^{*}=0$. Therefore in most circumstances we expect it will not be singular, and the asymptotic matching only requires $\mathbf{u}_{(2)}^{(+,+,-,-)}$to have leading order $(2 \varsigma)$.

As previously, we let $\mathbf{Y}=\mathbf{E}^{-1} \cdot \mathbf{V}$, with $\mathbf{Y}=(2 \varsigma)^{2} Y_{I}+(2 \varsigma) Y_{I I}+\ldots$, so that (118) simplifies to

$$
\begin{aligned}
(2 \varsigma)^{4} \frac{\partial \mathbf{Y}}{\partial \nu}=(2 \varsigma)^{4} \mathbf{D} \cdot \mathbf{Y}+(2 \varsigma)^{3} \mathbf{B}^{(1)} \cdot \mathbf{Y}+\mathbf{B}^{(2)} \cdot \mathbf{Y} \\
+(2 \varsigma)^{3} \sum_{1 \leq j, k \leq 3} \mathbf{B}_{(j, k)}^{(4)} \exp \left(\frac{\nu}{2}\left(d_{j j}+d_{k k}\right)\right),
\end{aligned}
$$

where $\mathbf{B}_{(j, k)}^{(4)}=-\mathbf{E}^{-1} \cdot \mathbf{A}_{(j, k)}^{(4)}$. The leading order solution is of the same form as (89), i.e.

$$
Y_{(I)}^{j}=K C^{j} \exp \left(d_{j j} \nu\right)
$$


for some constant $K$ to be determined further below. The asymptotic behaviour of the critical parameter is

$$
\begin{aligned}
\lambda^{\varsigma\left(\gamma_{2}^{*}, \gamma_{3}^{*}\right)} & =\lambda_{(I)}^{\left(\gamma_{2}^{*}, \gamma_{3}^{*}\right)}+(\varsigma)^{-1} \lambda_{(I I)}^{\left(\gamma_{2}^{*}, \gamma_{3}^{*}\right)}+\ldots \\
& =\lambda_{(I)}^{\left(\gamma_{2}^{*}, \gamma_{3}^{*}\right)}+(2 \varsigma)^{-1}\left(2 \lambda_{(I I)}^{\left(\gamma_{2}^{*}, \gamma_{3}^{*}\right)}\right)+\ldots
\end{aligned}
$$

We have already determined expressions for $\lambda_{(I)}^{\varsigma\left(\gamma_{2}^{*}, \gamma_{3}^{*}\right)}$ and $\lambda_{(I I)}^{\varsigma\left(\gamma_{2}^{*}, \gamma_{3}^{*}\right)}$ in Sections 5.2 and 5.3 .

The next order of (124) is

$$
\begin{aligned}
& \frac{\partial}{\partial \nu} Y_{(I I)}^{j}=d_{j j} Y_{(I I)}^{j}+K B_{j m}^{(1)} C^{m} \exp \left(\nu d_{m m}\right)+ \\
& K\left(2 \lambda_{(I I)}^{\left(\gamma_{2}^{*}, \gamma_{3}^{*}\right)} \frac{\partial d_{j j}}{\partial \lambda}+\nu \frac{\partial d_{j j}}{\partial R}\right) C^{j} \exp \left(\nu d_{j j}\right) \\
&+\sum_{1 \leq l, m \leq 3} B_{(l, m)}^{(4)}{ }^{j} \exp \left(\frac{\nu}{2}\left(d_{l l}+d_{m m}\right)\right) .
\end{aligned}
$$

We thus find that

$$
\begin{aligned}
Y_{(I I)}^{j}= & K B_{j m}^{(1)} C^{m}\left(d_{m m}-d_{j j}\right)^{-1} \exp \left(\nu d_{m m}\right)+ \\
& K C^{j}\left(B_{j j}^{(1)}+2 \lambda_{(I I)}^{\left(\gamma_{2}^{*}, \gamma_{3}^{*}\right)} \frac{\partial d_{j j}}{\partial \lambda}+\frac{\nu}{2} \frac{\partial d_{j j}}{\partial R}\right) \nu \exp \left(\nu d_{j j}\right)+C_{(I I)}^{j} \exp \left(\nu d_{j j}\right) \\
& +\sum_{1 \leq l, m \leq 3}\left(\frac{1}{2}\left(d_{l l}+d_{m m}\right)-d_{j j}\right)^{-1} B_{(l, m)}^{(4)}{ }^{j} \exp \left(\frac{\nu}{2}\left(d_{l l}+d_{m m}\right)\right) \\
& +\nu \sum_{a, b: a+b=2 j} B_{(a, b)}^{(4)}{ }^{j} \exp \left(\nu d_{j j}\right) \\
= & K B_{j m}^{(1)} C^{m}\left(d_{m m}-d_{j j}\right)^{-1}+\sum_{1 \leq l, m \leq 3}\left(\frac{1}{2}\left(d_{l l}+d_{m m}\right)-d_{j j}\right)^{-1} B_{(l, m)}^{(4)}{ }^{j} \\
& +C_{(I I)}^{j} \text { at } \nu=0 .
\end{aligned}
$$

Here $C_{(I I)}^{j}$ are undetermined constants and in the two summations we sum over all $l, m$ such that $d_{l l}+d_{m m} \neq 2 d_{j j}$.

We use the boundary condition to determine $K$. To implement the boundary condition we must consider the null-stress and null-displacement boundary conditions separately.

If there is a null-displacement boundary condition at $\partial \mathcal{B}^{*}$, then the $O((2 \varsigma))$ component of the null-displacement boundary condition is

$$
\begin{aligned}
K\left(E_{a k} B_{k m}^{(1)} C^{m}\left(d_{m m}-d_{k k}\right)^{-1}+2 \lambda_{(I I)}^{\left(\gamma_{2}^{*}, \gamma_{3}^{*}\right)} C^{k} \frac{\partial}{\partial \lambda} E_{a k}\right) & \\
& +\sum_{1 \leq l, m \leq 3}\left(\frac{1}{2}\left(d_{l l}+d_{m m}\right)-d_{k k}\right)^{-1} B_{(l, m)}^{(4)}{ }^{k} E_{a k}+q^{a}=0
\end{aligned}
$$

where $\mathbf{q}=\mathbf{Q} \cdot \mathbf{C}_{(I I)}, a \in\{1,2,3\}$ and we sum over all $l, m$ such that $d_{l l}+d_{m m} \neq$ 
$2 d_{j j}$. Here $\mathbf{Q}$ is the same as defined in Section 5.2. We dot multiply (131) with a vector perpendicular to the range of $\mathbf{Q}$ to solve for $K$.

Alternatively, if there is a stress boundary condition at $\partial \mathcal{B}^{*}$, then the boundary condition is implemented as follows. The $O\left((2 \varsigma)^{2}\right)$ of the displacement gradient is

$$
\left(\nabla \mathbf{u}_{(2)}\right)_{(I I)}=K \mathbf{r}+\mathbf{s},
$$

where for $a=2$ or 3 and $b \in\{1,2,3\}$,

$$
\begin{aligned}
r^{b}{ }_{1} & =2 \lambda_{(I I)}^{\left(\gamma_{2}^{*}, \gamma_{3}^{*}\right)} \frac{\partial}{\partial \lambda} E_{(b+3) j} C^{j}+\Gamma_{c 1}^{b} C^{j} E_{c j} \\
& +B_{j k}^{(1)} C^{k}\left(d_{k k}-d_{j j}\right)^{-1} E_{(b+3) j}+C_{(I I)}^{k} E_{(b+3) k} \\
r^{b}{ }_{a} & =2 i \gamma_{a}^{*} \lambda_{(I I)}^{\left(\gamma_{2}^{*}, \gamma_{3}^{*}\right)} \frac{\partial}{\partial \lambda} E_{b j} C^{j}+\Gamma_{c a}^{b} C^{j} E_{c j} \\
& +i \gamma_{a}^{*} B_{j k}^{(1)}\left(d_{k k}-d_{j j}\right)^{-1} C^{k} E_{b j}+i \gamma_{a}^{*} C_{(I I)}^{k} E_{b k} \\
s^{b}{ }_{1} & =\sum_{1 \leq j, l, m \leq 3} B_{(l, m)}^{(4)}{ }^{j} d_{j j}\left(\frac{1}{2}\left(d_{l l}+d_{m m}\right)-d_{j j}\right)^{-1} E_{b j}+C_{(I I)}^{j} E_{b(j+3)} \\
s^{b}{ }_{a} & =i \gamma_{a}^{*} \sum_{1 \leq l, m \leq 3} B_{(l, m)}^{(4)}{ }^{j}\left(\frac{1}{2}\left(d_{l l}+d_{m m}\right)-d_{j j}\right)^{-1} E_{b j}+i \gamma_{a}^{*} C_{(I I)}^{j} E_{b j} .
\end{aligned}
$$

In the summations, we sum over all $l, m$ such that $d_{l l}+d_{m m} \neq 2 d_{j j}$ and all $k \neq j$. The $O\left((2 \varsigma)^{2}\right)$ component of the stress boundary condition becomes

$$
\begin{aligned}
q^{a}+K\left(P_{c}^{a 1}{ }_{c} r_{d}{ }_{d}-\right. & \left.\frac{\Gamma_{0}}{\Gamma} \frac{\partial t^{a}}{\partial u^{b}} E_{b j} C_{j}+2 \lambda_{(I I)}^{\left(\gamma_{2}^{*}, \gamma_{3}^{*}\right)} \frac{\partial}{\partial \lambda} P^{a 1}{ }_{c} d \sum_{j=1}^{3} u_{(1, j)}^{*}{ }^{c} d\right) \\
& +P_{(2) c}^{a 1}{ }^{d} e^{f} \sum_{j, k=1}^{3} u_{(1, j)}^{*}{ }^{c}{ }_{d} u_{(1, k)}^{*}{ }_{f}{ }_{f}+P^{a 1}{ }_{c}{ }^{d} s^{c}{ }_{d}=0
\end{aligned}
$$

where $u_{(1, j)}^{*}$ is defined in (92)-(93), $\mathbf{q}=\mathbf{Q} \cdot \mathbf{C}_{(I I)}$ and $a \in\{1,2,3\}$. We dot multiply (137) with a vector perpendicular to the range of $\mathbf{Q}$ to solve for $K$.

\section{Boundary Layer Analysis of the Integrals Governing the Magni- tude of the Buckle}

In this section we determine the asymptotic order of the integrals governing the magnitude of the buckle (as defined in Section 4.2). However before doing this, we summarise our asymptotic analysis thus far. We must reappend the superscripts $(\alpha, \beta)$ to distinguish between the different Fourier modes. To leading order, the $k^{\prime}$ th component of $\mathbf{u}_{(1)}^{\left(\alpha \gamma_{2}, \beta \gamma_{3}\right)}$ (as defined in (23), and where $\alpha, \beta \in\{ \pm 1\})$ is equal to

$$
u_{(1)}^{\left(\alpha \gamma_{2}, \beta \gamma_{3}\right), k}=\sum_{j=1}^{3} C^{(\alpha, \beta) j} E_{k j}^{(\alpha, \beta)} \exp \left(\rho d_{j j}+i \varsigma\left(\gamma_{2}^{*} x^{2}+\gamma_{3}^{*} x^{3}\right)\right) .
$$


We have neglected terms of order $\varsigma^{-1} \exp \left(i \varsigma\left(\gamma_{2}^{*} x^{2}+\gamma_{3}^{*} x^{3}\right)\right)$ or lower. We define $\mathbf{E}^{(\alpha, \beta)}$ to be equal to $\mathbf{E}$ (as defined in (84)), except that we swap $\gamma_{2}^{*} \rightarrow \alpha \gamma_{2}^{*}$ and $\gamma_{3}^{*} \rightarrow \beta \gamma_{3}^{*}$ in the course of our determination of $\mathbf{E}$ throughout Section 5.2. We already have, by definition, that $\mathbf{E}^{(+,+)}=\mathbf{E}$. As was explained in Section 5.4, we find that $\mathbf{E}^{(-,+)}=\mathbf{E}^{(+,+)}$, except that (for all $\left.j\right) E_{2 j}^{(-,+)}=-E_{2 j}^{(+,+)}$and $E_{5 j}^{(-,+)}=-E_{5 j}^{(+,+)}$. Similarly, $\mathbf{E}^{(+,-)}=\mathbf{E}^{(+,+)}$, except that (for all $j$ ) $E_{3 j}^{(+,-)}=$ $-E_{3 j}^{(+,+)}$and $E_{6 j}^{(+,-)}=-E_{6 j}^{(+,+)}$. Finally, $\mathbf{E}^{(-,-)}=-\mathbf{E}^{(+,+)}$, except that (for all $j) E_{1 j}^{(-,-)}=E_{1 j}^{(+,+)}$and $E_{4 j}^{(-,-)}=E_{4 j}^{(+,+)}$. Through reference to (96) and the discussion of the structure of $\mathbf{Q}$ in Section 5.4 , we find that, $\mathbf{C}^{(\alpha, \beta)}=\mathbf{C}$ in the case of the null-displacement boundary condition (for all $\alpha$ and $\beta$ ). In the case of the null-stress boundary condition, $\mathbf{C}^{(+,+)}=\mathbf{C}$ (as defined in $\left.(96)\right), \mathbf{C}^{(-,+)}=$ $\left(C^{(+,+) 1},-C^{(+,+) 2}, C^{(+,+) 3}\right)^{T}, \mathbf{C}^{(+,-)}=\left(C^{(+,+) 1}, C^{(+,+) 2},-C^{(+,+) 3}\right)^{T}$ and $\mathbf{C}^{(-,-)}=\left(C^{(+,+) 1},-C^{(+,+) 2},-C^{(+,+) 3}\right)^{T}$.

The leading order of $\mathbf{u}_{(2)}$ is given by

$$
u_{(2)}^{(\alpha, \beta, \alpha, \beta), k}=(2 \varsigma)^{2} K^{(\alpha, \beta)} \sum_{j=1}^{3} C^{(\alpha, \beta) j} E_{k j}^{(\alpha, \beta)} \exp \left(2 \rho d_{j j}+2 i \varsigma\left(\gamma_{2}^{*} x^{2}+\gamma_{3}^{*} x^{3}\right)\right)
$$

where $\left.K^{(\alpha, \beta}\right)$ is determined from (131) or (137) depending on the boundary condition. Thus for example $K^{(-,+)}$may be determined by switching $\gamma_{2}^{*} \rightarrow-\gamma_{2}^{*}$ throughout the boundary layer analysis. As an aside, it may be observed that at $x_{c}^{1}, \mathbf{u}_{(2)}$ dominates $\mathbf{u}_{(1)}$ as $\varsigma \rightarrow \infty$, so that the buckled shape at the boundary layer is dominated by the second order solution (in the asymptotic limit). In general, if we were to continue solving for higher orders of $\mathbf{u}$, we would find that $\mathbf{u}_{(j)}$ dominates $\mathbf{u}_{(k)}$ for all $j>k$ at $x^{1}=x_{c}^{1}$ in the asymptotic limit.

We write the leading order of the displacement gradient over the boundary layer as

$$
\nabla \mathbf{u}_{(1)}^{\left(\alpha \gamma_{2}, \beta \gamma_{3}\right)} \simeq \varsigma \sum_{j=1}^{3} \mathbf{u}_{(1, j)}^{(*, \alpha, \beta)} \exp \left(\rho d_{j j}+i \varsigma\left(\gamma_{2}^{*} x^{2}+\gamma_{3}^{*} x^{3}\right)\right)
$$

where $\alpha, \beta \in\{ \pm 1\}$. Here $\mathbf{u}_{(1, j)}^{(*, \alpha, \beta)}$ is defined in (91), although we have appended the superscript $(\alpha, \beta)$ to distinguish between the different Fourier Modes. We determine the structure of $\mathbf{u}_{(1, j)}^{(*, \pm \alpha, \pm \beta)}$ using the above expressions for the structure of $\mathbf{E}^{(\alpha, \beta)}$ and $\mathbf{C}^{(\alpha, \beta)}$. Similarly,

$$
\nabla \mathbf{u}_{(2)}^{(\alpha, \beta, \alpha, \beta)}=(2 \varsigma)^{3} K^{(\alpha, \beta)} \sum_{j=1}^{3} \mathbf{u}_{(1, j)}^{(*, \alpha, \beta)} \exp \left(2 \rho d_{j j}+2 i \varsigma\left(\gamma_{2}^{*} x^{2}+\gamma_{3}^{*} x^{3}\right)\right),
$$

Recall that (as we noted in Section 6.1), if $\alpha \neq \mu$ and $\beta \neq \omega, \mathbf{u}_{(2)}^{(\alpha, \beta, \mu, \omega)}$ is of asymptotic order $O(\varsigma)$ and $\nabla \mathbf{u}_{(2)}^{(\alpha, \beta, \mu, \omega)}$ is of asymptotic order $O\left(\varsigma^{2}\right)$.

We determine the leading asymptotic orders of $g_{\alpha, \beta}^{\left(\gamma_{2}, \gamma_{3}\right)}$ and $h_{\alpha, \beta}^{\left(\gamma_{2}, \gamma_{3}\right)}$ as follows. We recall that these variables are each a sum of body and surface integrals. It 
can be seen from (53) that the body integrals are each of the form

$$
\int_{x_{c}^{1}}^{x_{+}^{1}} c\left(x^{1}\right) \exp (-\rho \text { Const }) d x^{1}
$$

where Const is a positive constant and $c$ is a function of $x^{1}$. It can be seen that the leading asymptotic order of (142) is

$$
\varsigma^{-1} c\left(x_{c}^{1}\right) \text { Const }^{-1} \text {. }
$$

It is clear that the surface integral over $\partial \mathcal{B}^{t}$ is asymptotically negligible because $\tau$ is independent of $\mathbf{F}$.

We are now ready to determine the leading asymptotic order of $g_{\alpha, \beta}^{\left(\gamma_{2}, \gamma_{3}\right)}$. Making use of (53) and (143), we thus have that

$$
g_{(\alpha, \beta)}^{\left(\gamma_{2}, \gamma_{3}\right)} \simeq \varsigma(2 \varsigma)^{3} a_{\alpha, \beta}^{\left(\gamma_{2}, \gamma_{3}\right)}\left(a_{-\alpha,-\beta}^{\left(\gamma_{2}, \gamma_{3}\right)}\right)^{2} \tilde{g}_{(\alpha, \beta)}^{\left(\gamma_{2}, \gamma_{3}\right)},
$$

where

$$
\begin{aligned}
& \tilde{g}_{(\alpha, \beta)}^{\left(\gamma_{2}, \gamma_{3}\right)} \simeq-\frac{1}{2}\left(4 x_{2}^{c} x_{3}^{c}\right) K^{(\alpha, \beta)} \operatorname{det}(\mathbf{g}) \times \\
& \sum_{j, k, l=1}^{3}\left(d_{j j}+d_{k k}+2 d_{l l}\right)^{-1} \frac{\partial^{3} \psi}{\partial \mathbf{F}^{3}}: \mathbf{u}_{(1, j)}^{\left(*, \alpha \gamma_{2}^{*}, \beta \gamma_{3}^{*}\right)}: \mathbf{u}_{(1, k)}^{\left(*, \alpha \gamma_{2}^{*}, \beta \gamma_{3}^{*}\right)}: \mathbf{u}_{(1, l)}^{\left(*,-\alpha \gamma_{2}^{*},-\beta \gamma_{3}^{*}\right)}
\end{aligned}
$$

where all of the expressions dependent on $x_{1}$ are evaluated at $x_{1}^{c}$.

We determine the leading asymptotic order of $h_{\alpha, \beta}^{\left(\gamma_{2}, \gamma_{3}\right)}$ to be

$$
h_{(\alpha, \beta)}^{\left(\gamma_{2}, \gamma_{3}\right)} \simeq \varsigma \tilde{h}_{(\alpha, \beta)}^{\left(\gamma_{2}, \gamma_{3}\right)}
$$

where

$$
\begin{aligned}
& \tilde{h}_{(\alpha, \beta)}^{\left(\gamma_{2}, \gamma_{3}\right)} \simeq-\frac{1}{2}\left(4 x_{2}^{c} x_{3}^{c}\right) \operatorname{det}(\mathbf{g}) \sum_{j, k=1}^{3}\left(d_{j j}+d_{k k}\right)^{-1} \times \\
& \left(\frac{\partial^{3} \psi}{\partial \mathbf{F}^{3}}: \nabla \mathbf{u}_{(2, p)}: \mathbf{u}_{(1, j)}^{(*,-\alpha,-\beta)}: \mathbf{u}_{(1, k)}^{(*, \alpha, \beta)}+\frac{\partial^{3} \psi}{\partial \mathbf{F}^{2} \partial \lambda}: \mathbf{u}_{(1, j)}^{(*,-\alpha,-\beta)}: \mathbf{u}_{(1, k)}^{(*, \alpha, \beta)}\right),
\end{aligned}
$$

and $\mathbf{n}$ is the unit outer normal. In the special case that (28) holds, we see from (61), (62), (145) and (147) that

$$
a_{\alpha, \beta}^{\left(\varsigma \gamma_{2}^{*}, \gamma_{3}^{*}\right)} a_{-\alpha,-\beta}^{\left(\varsigma \gamma_{2}^{*}, \varsigma \gamma_{3}^{*}\right)}=\left|a_{\alpha, \beta}^{\left(\varsigma \gamma_{2}^{*}, \varsigma \gamma_{3}^{*}\right)}\right|^{2} \simeq-\frac{1}{4} \frac{\tilde{h}_{(\alpha, \beta)}^{\left(\gamma_{2}, \gamma_{3}\right)}}{\tilde{g}_{(\alpha, \beta)}^{\left(\gamma_{2}, \gamma_{3}\right)}} \varsigma^{-3} \lambda_{(2)} .
$$

We thus see that the magnitude of the buckle has leading asymptotic order $\zeta^{-\frac{3}{2}} \lambda_{(2)}^{\frac{1}{2}}$. 


\section{An Application: A Compressed NeoHookean Annulus}

We apply the preceding theory to the following two-dimensional problem. We consider an annulus, with internal radius $R_{0}$ and external radius $R_{1}$ (we scale $R_{1}$ to be 1 ). We work in polar coordinates, with reference coordinates $\{R, \Theta\}$ and current coordinates $\{r, \theta\}$. For convenience, we consider the deformation gradient to be relative to the orthonormal basis vectors $\left(\mathbf{e}_{R}, \mathbf{e}_{\Theta}\right)$, so that there is no distinction between contravariant and covariant components. The ring is modelled as an incompressible NeoHookean Material, with strain energy density given by

$$
\Psi(\mathbf{F})=\frac{1}{2} \Sigma \mathbf{F}: \mathbf{F},
$$

where $\Sigma$ is the shear modulus (which is scaled to be 1). Since the ring is incompressible, we must include an extra term to enfore the incompressibility constraint

$$
J-1=0
$$

so that $\hat{\Psi}=\Psi-p(J-1)$. We have outlined our method for incompressible elasticity in the extra supplementary material. The Piola-Kirchhoff Stress is therefore $\mathbf{P}=\Sigma \mathbf{F}-p J F^{-T}$, and the equation of equilibrium is $-\nabla \cdot \mathbf{P}=\mathbf{0}$. The ring is unstressed at $R_{0}$. At $R_{1}$, the displacement is stipulated to be $-v \mathbf{e}_{R}$ (for $v \geq 0$ ). The boundary conditions are thus

$$
\begin{aligned}
\mathbf{P} \cdot \mathbf{N} & =\mathbf{0} \text { at } R=R_{0} \text { and } \\
\mathbf{u} & =-v \mathbf{e}_{R} \text { at } R=R_{1} .
\end{aligned}
$$

The critical parameter, i.e. the displacement $(v)$, is gradually increased until the system buckles. The base solution is stipulated to be radially symmetric (so that $\theta=\Theta$ ). We obtain the base solution as follows. The incompressibility constraint yields the ordinary differential equation $\frac{\partial r}{\partial R} \times \frac{r}{R}-1=0$. We find that $r^{2}=R^{2}+C$, for some constant $C$. In the base state, we have $\mathbf{F}=$ $F_{R R} \mathbf{e}_{R} \otimes \mathbf{e}_{R}+F_{\Theta \Theta} \mathbf{e}_{\Theta} \otimes \mathbf{e}_{\Theta}$, where $F_{R R}=\frac{\partial r}{\partial R}$ and $F_{\Theta \Theta}=r / R$. The equation of mechanical equilibrium yields an ordinary differential equation of the form

$$
\Sigma \frac{\partial^{2} r}{\partial R^{2}}+\frac{\Sigma+p_{(0)}}{R}\left(\frac{\partial r}{\partial R}-\frac{r}{R}\right)-\frac{\partial p_{(0)}}{\partial R} \frac{\partial R}{\partial r}-p_{(0)} \frac{\partial}{\partial R}\left(F_{\Theta \Theta}\right)=0 .
$$

Through the displacement boundary condition at $R_{1}$, we find that $C=\left(R_{1}-\right.$ $v)^{2}-R_{1}^{2}$. We may then determine $p_{(0)}$ by integrating (153) from $R_{0}$ to $R_{1}$.

The incremental equations governing the stability and postbuckling behaviour are implemented according to the methodology outlined in the electronic supplementary material. We note that the fixed-reference elastic moduli have the form $P^{a B}{ }_{c}{ }^{D}=\Sigma \delta^{a}{ }_{c} \delta^{B}{ }_{D}$. The higher order fixed-reference elastic moduli are zero. The critical value of $v$ when mode $n$ buckles is $v^{(n)}$. In brief, the buckled 
expansion is

$$
\begin{aligned}
& v=v^{(n)}+\frac{1}{2} \epsilon^{2} v_{(2)}+\ldots \\
& \mathbf{u}=\mathbf{u}_{(0)}+\epsilon \mathbf{u}_{(1)}+\frac{1}{2} \epsilon^{2} \mathbf{u}_{(2)}+\ldots \\
& p=p_{(0)}+\epsilon p_{(1)}+\frac{1}{2} \epsilon^{2} p_{(2)}+\ldots
\end{aligned}
$$

In brief, we have that

$$
\begin{aligned}
u_{(1)}= & \left(a_{+} Z^{(n) R} \exp (i n \Theta)+a_{-} Z^{(-n) R} \exp (-i n \Theta)\right) \mathbf{e}_{R} \\
& +\left(a_{+} Z^{(n) \Theta} \exp (i n \Theta)+a_{-} Z^{(-n) \Theta} \exp (-i n \Theta)\right) \mathbf{e}_{\Theta}, \\
p_{(1)}= & a_{+} Z^{(n) p} \exp (i n \Theta)+a_{-} Z^{(-n) p} \exp (-i n \Theta),
\end{aligned}
$$

where $\mathbf{e}_{R}$ and $\mathbf{e}_{\Theta}$ are the orthonormal cylindrical basis vectors. It may be easily shown ${ }^{7}$ that we may assume without loss of generality that each of $Z^{(n) R}, Z^{(n) p}$, $Z^{(-n) R}$ and $Z^{(-n) p}$ are real, and $Z^{(n) \Theta}$ and $Z_{(2)}^{(-n) \Theta}$ are purely imaginary. Since $a_{+}=\bar{a}_{-}$and $Z^{(-n)}=\bar{Z}^{(n)}$, we may thus write the solution in the form

$$
\begin{aligned}
& u_{(1)}=a Z^{(n, *) R} \cos (n \Theta) \mathbf{e}_{R}+a Z^{(n, *) \Theta} \sin (n \Theta) \mathbf{e}_{\Theta}, \\
& p_{(1)}=a Z^{(n, *) p} \cos (n \Theta),
\end{aligned}
$$

where $\mathbf{Z}^{(n, *)}$ and $a$ are real. We similarly find that we may write the $O\left(\epsilon^{2}\right)$ solution in the form $\mathbf{u}_{(2)}=v_{(2)} \mathbf{u}_{(2, p)}+a^{2} \mathbf{u}_{(2, a)}$ (similarly for $p_{(2)}$ ), where

$$
\begin{aligned}
& u_{(2, a)}=\left(Z_{(2)}^{(2 n, *) R} \cos (2 n \Theta)+Z_{(2)}^{(0, *)}\right) \mathbf{e}_{R}+Z_{(2)}^{(2 n, *) \Theta} \sin (2 n \Theta) \mathbf{e}_{\Theta} \\
& p_{(2, a)}=Z_{(2)}^{(2 n, *) p} \cos (2 n \Theta)+Z_{(2)}^{(0, *) p}
\end{aligned}
$$

and $\mathbf{Z}_{(2)}^{(2 n, *)}$ and $Z_{(0)}^{(2 n, *)}$ are real functions of $R$. The above variables (and $\mathbf{u}_{(2, p)}$ ) may be solved for using the method outlined in the electronic supplementary materials.

The ordinary differential equations governing the above variables are highly unstable for large $n$. This is not surprising because our boundary layer analysis indicates that the solutions are of the order of $\exp (n \rho)$. The determinantal method (whereby one separately integrates two independent solutions and superposes them) is therefore unsuitable (Goriely et al. [12]). We thus employ the method of compound matrices. This has been previously done in the numerical solution of the incremental equations of nonlinear elasticity by (amongst others) Haughton and Orr [13], Goriely et al. [12] and the authors of this paper [19]. The method is sourced from Ng and Reid [25], to which the reader is referred for a more detailed explanation. 

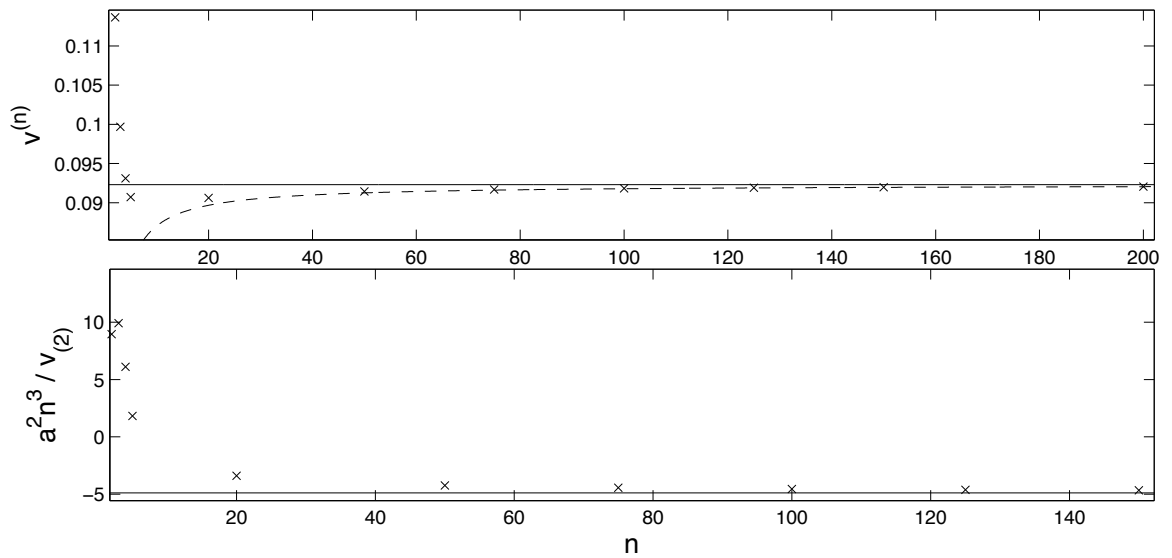

Figure 1: At the top we plot the asymptotic convergence of the critical displacement, with the limit $v_{(I)}$ plotted in a solid line and $v_{(I)}+\varsigma^{-1} v_{(I I)}^{(1)}$ plotted in a dashed line. The dimensions are such that $R_{0} / R_{1}=1 / 2$. At the bottom we plot the asymptotic convergence of the magnitude $|a|$ of the buckle. The asymptotic limit is plotted in a solid line. The critical modes are $\{2,3,4,5,20,50,75,100,125,150,200\}$.
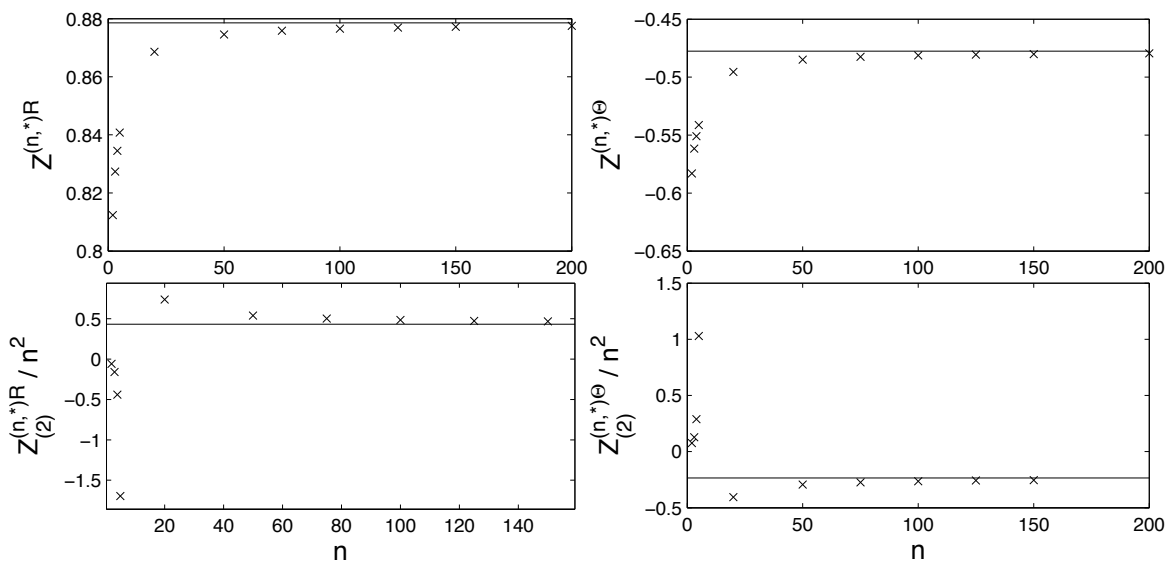

Figure 2: The asymptotic convergence of the first and second order components of the displacement as the wavenumber $n$ converges to infinity. Here $R_{0} / R_{1}=1 / 2$ and we employ the displacement boundary condition at $R_{1}$. The variables are evaluated at $R_{0}$. The asymptotic limits are plotted in solid lines. 

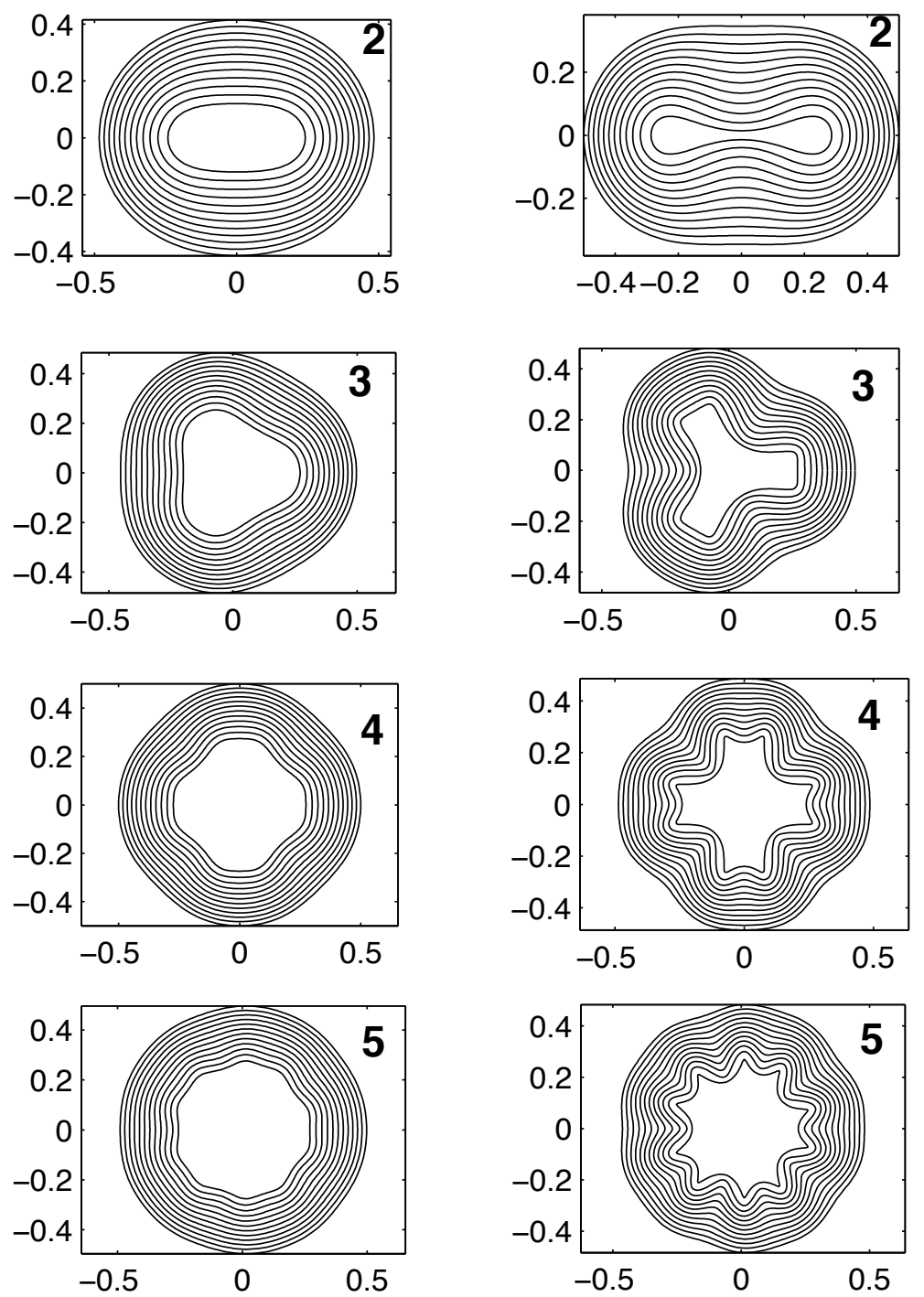

Figure 3: The postbuckled profiles for modes 2-5. We employed the displacement boundary condition and $R_{0} / R_{1}=0.5$. We have plotted equally-spaced contours of $R$ (the reference coordinate) from 0.5 to 0.65 . In the first column $\epsilon^{2} v_{(2)}=0.005$, and in the second column $\epsilon^{2} v_{(2)}=0.025$. 

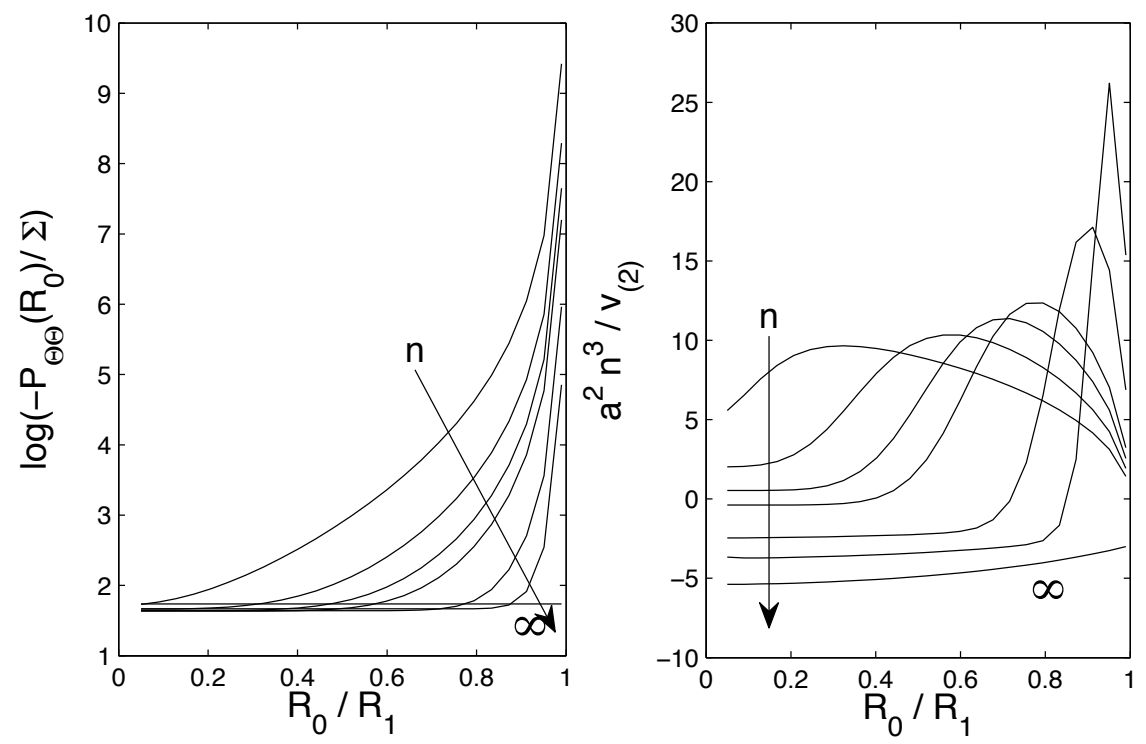

Figure 4: The effect of varying the ratio $R_{0} / R_{1}$ on the onset of buckling, for modes $n \in$ $\{2,3,4,5,10,20\}$, and the asymptotic limit $n=\infty$. On the left we plot the logarithm of the hoop stress at $R_{0}$ at the time of buckling, and on the right we plot the magnitude $a$ of the buckle. Online Version in Colour

\subsection{Results}

We test the accuracy of our ansatz by comparing it to the direct numerical solution of the incremental equations. The asymptotic convergence for large $n$ is plotted in Figures 1 and 2. Here $R_{0} / R_{1}=1 / 2$. It is interesting to observe that for low modes, the buckle is supercritical and stable (since $a>0$ ), but for higher modes (and in the asymptotic limit), $a<0$, so that the buckle is subcritical and unstable (refer to Section 4.3 for an explanation of this analysis). Furthermore the first mode to go unstable appears to be between 5 and 20. Observe also that the asymptotic convergence of $\mathbf{Z}_{(2)}^{n, *}$ appears to be much slower than the asymptotic convergence of $\mathbf{Z}_{(1)}^{n, *}$. This is not surprising because we needed to solve an additional order of the asymptotic equations to determine $\mathbf{Z}_{(2)}^{n, *}$.

We plot the buckled contours in Figure 3 to demonstrate the qualitative nature of the wrinkling. We plot the buckled contours for modes 2-5, with $\epsilon^{2} v_{(2)}=0.005$ and $\epsilon^{2} v_{(2)}=0.025$. These plots demonstrate the emergence of the boundary layer. It may be observed that as the mode number $n$ increases, the displacement is increasingly concentrated at $R_{0}$. Furthermore, consistent with our boundary layer analysis, for fixed $\epsilon^{2} v_{(2)}$, as $n$ increases the buckled profile is increasingly dominated by $\mathbf{u}_{(2)}$ at the expense of $\mathbf{u}_{(1)}$. In particular, with the $R=R_{0}$ contour when $\epsilon^{2} v_{(2)}=0.025$, it may be seen that the mode 2 plot is dominated by $\mathbf{u}_{(1)}$ (as the wrinkled shape is predominantly of mode 2 ), but the mode 5 plot is dominated by $\mathbf{u}_{(2)}$ (the wrinkled shape is predominantly of mode 10).

In Figure 4 we investigate the dependence of the wrinkling on the ratio

\footnotetext{
${ }^{7}$ See MacLaurin et al. [19] for a more detailed explanation.
} 
$R_{0} / R_{1}$. It may be seen that the asymptotic limit of the hoop stress $P_{\Theta \Theta}$ at $R_{0}$ at the time of buckling is independent of $R_{0} / R_{1}$. Furthermore our results suggest that as $R_{0} / R_{1} \rightarrow 1$, the mode which buckles first asymptotes to $\infty$. We also see that the magnitude $a$ of all of the buckles gets smaller as $R_{0} / R_{1} \rightarrow 1$. However in the asymptotic limit, $a$ is always less than zero, meaning that in the asymptotic limit the wrinkles are eventually subcritical.

\section{Discussion}

In these results we have seen that the asymptotic limit $v_{(I)}$ was never the first mode to go unstable. Moulton and Goriely [21] observed similar results to ours in their various models of an externally compressed tube (without growth): the asymptotic limit of the critical pressure appears to occur well after the initial critical pressure. However when the bifurcation parameter was some form of growth $^{8}$, the asymptotic limit of the critical growths of the modes was close to the critical growth of the initial mode to go unstable (Moulton and Goriely [21]). The authors observed a similar phenomenon in their modelling of the growthinduced buckling of tumour capillaries [19]. They proved using the methods of this paper that sometimes the asymptotic limit was the infimum of the critical growths. This phenomenon arises because the (confined) growth generates an enormous hoop stress at the inner boundary. It underscores our discussion in Section 4 that we cannot simply assume that the system adopts the first mode to go unstable: such a mode may not even exist, or even if it does exist, then it may have such a fine wavelength that it lies outside the validity of the model. Furthermore we expect that such fine wrinkled patterns are vulnerable to inhomogeneities, so that it is difficult to predict from analytic consideration of the model the preferred buckled mode of the system. Perhaps these considerations suggest the importance of performing an imperfection sensitivity analysis in the manner of Cao and Hutchinson [4].

It is possible that our analysis might shed some light on the period-doubling phenomena observed in (for example) Brau et al. [2] and Li et al. [17]. In these phenomena, in general, an initial wrinkled pattern was observed, which over time evolved into a pattern with double the wavelength. It is possible that, $\lambda_{(I)}^{\left(\gamma_{2}^{*}, \gamma_{3}^{*}\right)}$ is close to the infimum of the points of instability of the modes, which would explain the susceptibility to fine wrinkling. However as $\lambda$ increases, modes of higher wavelength become unstable and eventually more energetically favourable, so that the system eventually jumps to these preferred modes.

Finally we note that many of the phenomena outlined in the introduction involve the wrinkling of a thin film anchored to some sort of compliant substrate (for example Cai et al. [3], Hohlfeld and Mahadevan [14], Cao and Hutchinson [5]). If the film is sufficiently thin, we would not expect the boundary layer analysis of this paper to be accurate. However it is possible that the method of this paper could be generalised into a two (or one) dimensional model of the film, coupled with a boundary layer analysis of the substrate. ${ }^{9}$

\footnotetext{
${ }^{8}$ Using the modelling briefly outlined in Section 5.1, with no external compression.

${ }^{9}$ The cited works have already gone some way towards this.
} 


\section{Conclusion}

In this paper we have investigated surface-wrinkling in elastostatic problems. We determined the onset of buckling using a linear-stability analysis, and the leading-order postbuckling behaviour through consideration of higher-order terms of the energy. We then applied a boundary-layer analysis to this problem, determining the leading order behaviours of the eigenmode, the critical parameter, and the magnitude of the buckle. We found that the magnitude of a buckle with wavenumbers $\varsigma \gamma_{2}^{*}$ and $\varsigma \gamma_{3}^{*}$ (for fixed $\gamma_{2}^{*}$ and $\gamma_{3}^{*}$ ) has leading asymptotic order $\varsigma^{-\frac{3}{2}} \sqrt{\lambda_{(2)}}$, for an increment $\lambda_{(2)}$ of the critical parameter beyond the critical time of buckling. We finally confirmed the accuracy of our ansatz on a compressed NeoHookean ring.

\section{Acknowledgements}

James MacLaurin wishes to thank the University of Oxford and family for funding. He also thanks his DPHIL examiners, Prof. Alain Goriely and Prof. Mark Chaplain, and Dr Derek Moulton for useful discussions. Dr Tiina Roose is supported by a Royal Society University Research Fellowship.

\section{References}

[1] M.A. Biot. Fundamental skin effect in anisotropic solid mechanics. International Journal of Solids and Structures, 2:645-663, 1966

[2] Fabian Brau, Hugues Vandeparre, Abbas Sabbah, Christophe Poulard, Arezki Boudaoud, and Pascal Damman. Multiple-length-scale elastic instability mimics parametric resonance of nonlinear oscillators. Nature Physics, 7:56-60, 2011.

[3] S. Cai, D. Breid, A.J. Crosby, Z. Suo, and J.W. Hutchinson. Periodic patterns and energy states of buckled film on compliant surfaces. Journal of the Mechanics and Physics of Solids, 59:1094-1114, 2011.

[4] Y. Cao and J.W. Hutchinson. From wrinkles to creases in elastomers: the instability and imperfection-sensitivity of wrinkling. Proceedings of the Royal Society A, 468:94-115, 2012.

[5] Yanping Cao and John W. Hutchinson. Wrinkling phenomena in neohookean film / substrate bilayers. Transactions of the ASME, 79, 2012.

[6] Ciprian Coman. Global asymptotic approximations for wrinkling of polar orthotropic annular plates in tension. International Journal of Solids and Structures, 47:1572-1579, 2010.

[7] Ciprian D. Coman and Andrew Bassom. On the wrinkling of a pre-stressed annular thin film in tension. Journal of the Mechanics and Physics of Solids, $55: 1601-1617,2007$.

[8] Julien Dervaux and Martine Ben Amar. Buckling condensation in constrained growth. Journal of the Mechanics and Physics of Solids, 59:538560, 2011. 
[9] Michel Destrade, Aisling Ni Annaidh, and Ciprian D. Coman. Bending instabilities of soft biological tissues. International Journal of Solids and Structures, 46:4322-4330, 2009.

[10] Lawrence Evans. Partial Differential Equations. American Mathematical Society, 1998.

[11] Alain Goriely, Mark Robertson-Tessi, Michael Tabor, and Rebecca Vandiver. Elastic growth models. 2008.

[12] Alain Goriely, R. Vandiver, and M.Destrade. Nonlinear euler buckling. Proceedings of the Royal Society A, 464:3003-3019, 2008.

[13] D. Haughton and A. Orr. On the eversion of compressible elastic cylinders. International Journal of Solids and Structures, 34:1893-1914, 1997.

[14] Evan Hohlfeld and L. Mahadevan. Unfolding the sulcus. Physical Review Letters, 106, 2011.

[15] W.T. Koiter. Elastic Stability of Solids and Structures. Cambridge University Press, 2009.

[16] Bo Li, Yan-Ping Cao, Xi-Qiao Feng, and Huajian Gao. Surface wrinkling of mucosa induced by volumetric growth: Theory, simulation and experiment. Journal of the Mechanics and Physics of Solids, 59:758-774, 2011.

[17] Bo Li, Fei Jia, Yan-Ping Cao, Xi-Quiao Feng, and Huajian Gao. Surface wrinkling patterns on a core-shell soft sphere. Physical Review Letters, $106,2011$.

[18] James MacLaurin. The buckling of capillaries in tumours. PhD thesis, University of Oxford, 2012.

[19] James MacLaurin, Jon Chapman, Gareth Wyn Jones, and Tiina Roose. The buckling of capillaries in solid tumours. Proceedings of the Royal Society A, 2012.

[20] Jerrold E. Marsden and Thomas J.R. Hughes. Mathematical Foundations of Elasticity. Dover, 1983.

[21] Derek Moulton and Alain Goriely. Circumferential buckling instability of a growing cylindrical tube. Journal of the Mechanics and Physics of Solids, 59:525?537, 2011.

[22] Derek Moulton and Alain Goriely. Possible role of differential growth in airway wall remodeling in asthma. Journal of Applied Physiology, 110: 1003-1012, 2011.

[23] Pablo V. Negron-Marrero and Errol Montes-Pizarro. The complementing condition and its role in a bifurcation theory applicable to nonlinear elasticity. New York Journal of Mathematics, 17a:1-21, 2011.

[24] Pablo V. Negron-Marrero and Errol Montes-Pizarro. Violation of the complementing condition and local bifurcation in nonlinear elasticity. Journal of Elasticity, 107:151-178, 2012. 
[25] B.S. Ng and W.H. Reid. The compound matrix method for ordinary differential systems. Journal of Computational Physics, 58:209-228, 1985.

[26] R. Ogden. Non-Linear Elastic Deformations. Ellis Horwood Limited, 1984.

[27] Edward Rodriguez, Anne Hoger, and Andrew D. McCulloch. Stressdependent finite growth in soft elastic tissues. Journal of Biomechanics, $27: 455-467,1994$.

[28] A.S.D Wang and A. Ertepinar. Stability and vibrations of elastic thickwalled cylindrical and spherical shells subjected to pressure. International Journal of Nonlinear Mechanics, pages 539-555, 1972. 